\title{
THE INTERACTION OF ASYMPTOTIC GIANT BRANCH STARS WITH THE INTERSTELLAR MEDIUM
}

\author{
Eva Villaver ${ }^{1}$, Arturo Manchado ${ }^{2,3}$, and Guillermo García-Segura ${ }^{4}$ \\ ${ }^{1}$ Departamento de Física Teórica, Universidad Autónoma de Madrid, Cantoblanco 28049 Madrid, Spain; eva.villaver@uam.es \\ 2 Instituto de Astrofísica de Canarias, Vía Láctea S/N, E-38200 La Laguna, Tenerife, Spain \\ ${ }^{3}$ Consejo Superior de Investigaciones Científicas, Spain; amt@11.iac.es \\ ${ }^{4}$ Instituto de Astronomía-UNAM, Apartado postal 877, Ensenada, 22800 Baja California, Mexico; ggs@astrosen.unam.mx \\ Received 2011 October 7; accepted 2012 January 9; published 2012 March 13
}

\begin{abstract}
We study the hydrodynamical behavior of the gas expelled by moving asymptotic giant branch stars interacting with the interstellar medium (ISM). Our models follow the wind modulations prescribed by stellar evolution calculations, and we cover a range of expected relative velocities (10-100 $\mathrm{km} \mathrm{s}^{-1}$ ), ISM densities (between 0.01 and $1 \mathrm{~cm}^{-3}$ ), and stellar progenitor masses $\left(1\right.$ and $\left.3.5 M_{\odot}\right)$. We show how and when bow shocks and cometary-like structures form, and in which regime the shells are subject to instabilities. Finally, we analyze the results of the simulations in terms of the different kinematical stellar populations expected in the Galaxy.
\end{abstract}

Key words: hydrodynamics - ISM: kinematics and dynamics - ISM: structure - stars: AGB and post-AGB - stars: winds, outflows

Online-only material: animations, color figures

\section{INTRODUCTION}

At the end of their lives low- and intermediate-mass stars (those with main-sequence masses between 1 and $8 M_{\odot}$ ) ascend the asymptotic giant branch (AGB) in the H-R diagram, where one of the most remarkable characteristics of their evolution is the ejection of the stellar envelope in a series of high mass-loss rate events during the thermal-pulsing phase at the end of the AGB stage.

While evolving, stars move within the gravitational field of the galaxy, and AGB stars are no exception. The association of a star with the different galactic components determines on a first order its velocity within the Galaxy. This stellar motion influences the structure and dynamics of the ejected AGB envelope as the stellar mass loss interacts with the local interstellar medium (ISM; Villaver et al. 2003). Moreover, mostly due to ram pressure stripping, an important fraction of the mass ejected by the star along the AGB is left downstream of the motion, forming cometary-like structures behind the star, even when low-velocity interactions are considered (see, e.g., Villaver et al. 2003).

The striking observations of the Mira AB binary system (Martin et al. 2007) confirmed this theoretical scenario, revealing a surrounding arc-like structure and a stream of material stretching $2^{\circ}$ away in opposition to the arc. Recently, more AGB stars showing the effects of the interaction with the ISM in their circumstellar envelopes (CSEs) have been found (e.g., Libert et al. 2008, 2009, 2010; Matthews \& Reid 2007; Ueta et al. 2010; Jorissen et al. 2011).

AGB stars eventually become planetary nebulae (PNe), and as the stellar effective temperature increases, the AGB envelope becomes ionized. In PNe, the asymmetries developed as a consequence of the interaction process with the ISM are in many cases a major morphological feature (see, e.g., Tweedy \& Kwitter 1996; Xilouris et al. 1996; Borkowski et al. 1993; Tweedy et al. 1995; Soker \& Zucker 1997; Guerrero et al. 1998; Chu et al. 2009; Ramos-Larios \& Phillips 2009; Ransom et al. 2008; Szentgyorgyi et al. 2003; López et al. 2011).

Gurzadyan (1969) was the first to suggest the interaction of the PN with the ISM as a possible mechanism to explain the observed asymmetries. The first theoretical studies (see, e.g., Smith 1976; Isaacmann 1979; Borkowski et al. 1990; Soker et al. 1991) arrived at the conclusion that the nebula fades away before any disruption of the nebular shell becomes noticeable unless high relative velocities or densities were involved. But these studies only considered the interaction process once the nebular shell was already formed. In Villaver et al. (2003), by studying the interaction process as the star evolves along the AGB phase, we demonstrated that the star-ISM interaction can be predominant even at low ISM densities and/or velocities, given that it appears as a direct consequence of evolution of the evolving AGB winds. Other models to study the interaction process in PN shells have been published since then to explain the morphologies observed in individual objects (Szentgyorgyi et al. 2003; Villaver \& Stanghellini 2005; Wareing et al. 2006, 2007b). In particular, the detailed observations of the Mira cometary structure (Martin et al. 2007) have motivated a wealth of elaborated theoretical work of the interaction (Wareing et al. 2007c; Raga et al. 2008; Raga \& Cantó 2008; Esquivel et al. 2010), with a stellar wind and ISM parameters chosen to reproduce the observations of this particular object.

Raga \& Cantó (2008) presented analytical predictions for the velocity of the material in the wake of Mira, as a function of the distance to the stellar source, and compared with the $21 \mathrm{~cm}$ observations of the system. Wareing et al. (2007c) studied numerically the interaction between the Mira wind and the ISM by using an isotropic and constant AGB wind with a mass-loss rate of $3 \times 10^{-7} M_{\odot} \mathrm{yr}^{-1}$ (variations of the mass loss by factors of three times this value were also considered) and a velocity of $5 \mathrm{~km} \mathrm{~s}^{-1}$ in a three-dimensional grid in order to match the overall observed structure. A three-dimensional domain was adopted as well by Raga et al. (2008) with similar parameters for the constant AGB wind $\left(7.7 \times 10^{-7} M_{\odot} \mathrm{yr}^{-1}\right.$ and $10 \mathrm{~km} \mathrm{~s}^{-1}$ for the mass loss and velocity, respectively) as those used by Wareing et al. (2007c). But in this case, with the goal of reproducing the double-shock structure observed in the cometary head, a dependency with latitude is set for the stellar wind. The two-dimensional adaptive mesh refinement work of Esquivel et al. (2010) aims to reproduce the broad-head narrow-tail structure observations of Mira that previous simulations (Wareing 
et al. 2007c; Raga et al. 2008) failed to reproduce. For that, they considered a changing ISM environment (a dense ISM turns into a low-density rarefied medium with the conditions of the local bubble) and used the same wind parameters as those adopted by Wareing et al. (2007c). What all these numerical simulations have in common is that the wind and ISM parameters have been chosen to reproduce the observations of a particular object.

A more general case of the interaction under a broader range of conditions has been explored in the three-dimensional simulations by Wareing et al. (2007a). Wareing et al. (2007a) examined a range of velocities for the interaction between 25 and $125 \mathrm{~km} \mathrm{~s}^{-1}$. However, the AGB wind was assumed to be constant within each simulation. Four different values of the AGB mass-loss rates were explored: $5 \times 10^{-7}$ (for a relative velocity of $25 \mathrm{~km} \mathrm{~s}^{-1}$ through an ISM with $\left.0.01 \mathrm{~cm}^{-3}\right), 5 \times$ $10^{-6}$ (for 50,75 , and $125 \mathrm{~km} \mathrm{~s}^{-1}$ interactions), and $10^{-7}$ (for a relative velocity of $100 \mathrm{~km} \mathrm{~s}^{-1}$ ) under ISM densities of $2 \mathrm{~cm}^{-3}$. The temperature of the stellar wind is set at $10^{4} \mathrm{~K}$, which is the lowest value for which the cooling function is defined in their simulation.

It is well known observationally that the mass loss experienced by AGB stars is not constant, and it has been shown that wind variations associated with the thermal pulses can lead to the formation of multiple shell structures and large haloes around AGB stars (see, e.g., Villaver et al. 2002a, 2002b; Schönberner et al. 2005). While realistic variable mass-loss rates along the AGB phase were explored in the study of the interaction process between an AGB star and the ISM (Villaver et al. 2003; Szentgyorgyi et al. 2003; Villaver \& Stanghellini 2005), we still lack a systematic study covering the range of expected relative velocities and ISM densities that follows the evolution of the mass loss from the star for different progenitors. This is precisely the goal of the present study. In this paper, we present simulations of the formation of extended shells along the AGB phase and their interaction with the ISM under a wide range of conditions. In our models, mass loss is not a free parameter, but follows the stellar evolution prescriptions. This study is motivated by the wealth of new observations of the resolved extended structure around AGB stars that are becoming readily available from the Herschel Space Observatory (Ladjal et al. 2010; Mayer et al. 2011; Jorissen et al. 2011) and will be complemented in the near future with $A L M A$.

The paper is organized as follows: in Section 2, we provide the details of the numerical method, the initial and boundary conditions, including a description of the parameters used to characterize the environment, and the stellar dynamics; in Section 3, we describe the effects of the different ISM conditions during the early phase of the evolution of the AGB when the stellar wind is constant; in Section 4, we describe the simulations by showing the evolution of the shells formed as the star ascends the AGB phase under different conditions; in Section 5, we discuss the development of instabilities in the shells; in Section 6, we discuss the effect of using different progenitor masses on the interaction; and in Sections 7 and 8, we present, respectively, a general discussion of the results and the conclusions of this work.

\section{THE NUMERICAL METHOD: INITIAL AND BOUNDARY CONDITIONS}

We have performed numerical simulations with the fluid solver ZEUS-3D (Stone \& Norman 1992a, 1992b; Stone et al. 1992), developed by M. L. Norman and the Laboratory for Computational Astrophysics. The computations have been carried

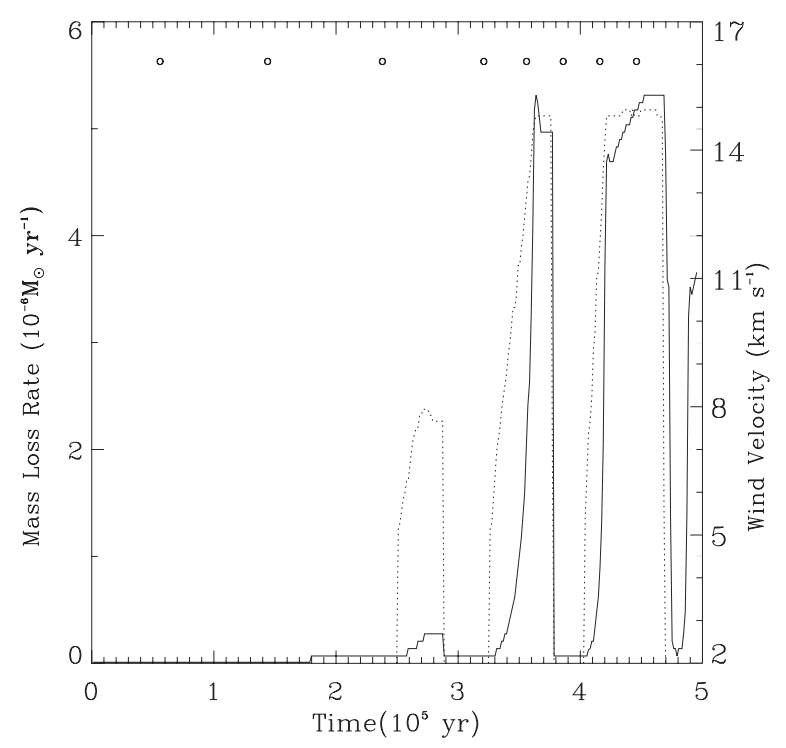

Figure 1. Mass-loss rate and wind expansion velocity used in the simulations of the $1 M_{\odot}$ star along the AGB phase. The solid line shows the mass-loss rate $\left(10^{-6} M_{\odot} \mathrm{yr}^{-1}\right.$, left scale), and the dotted line shows the terminal wind velocity ( $\mathrm{km} \mathrm{s}^{-1}$, right scale). The lowest mass-loss rates, not easily visible at the scale of the plot, are in the range $(1-0.6) \times 10^{-8} M_{\odot} \mathrm{yr}^{-1}$ with velocities between 2 and $5 \mathrm{~km} \mathrm{~s}^{-1}$. The circles in the top part of the plot mark the time at which the outputs of Figure 4 are shown.

out on a two-dimensional spherical polar grid with the angular coordinate ranging from $0^{\circ}$ to $180^{\circ}$ and a physical radial extension of $4 \mathrm{pc}$. The simulations have resolutions of $800 \times 720$ zones in the radial and angular coordinates of the grid, respectively, but a few models at lower resolutions $(400 \times 360$ radial and angular coordinates, respectively) have also been computed. The models include the Raymond \& Smith (1977) cooling curve above $10^{4} \mathrm{~K}$. For temperatures below $10^{4} \mathrm{~K}$ the gas is allowed to cool down with the radiative cooling curves given by Dalgarno \& McCray (1972) and MacDonald \& Bailey (1981).

Our boundary conditions are the AGB stellar wind and the parameters that define the physics of the ISM. The evolution of the star along the AGB phase is followed by feeding continuously the center of the grid with the stellar wind. The mass loss and wind temperature and velocity during the AGB phase have been taken from Vassiliadis \& Wood (1993). The wind temperature is assumed to be the effective temperature of the star. The simulations start at the early-AGB phase, before the onset of the first thermal pulse, and continue until the end of the AGB phase. Further details of the wind assumptions and gas evolution in a static configuration for different conditions can be found in Villaver et al. (2002a).

To study the effect of the interaction on different progenitors, we have used stellar models for 1 and $3.5 M_{\odot}$ stars (mainsequence masses). Figure 1 shows the mass loss (left axis, solid line) and stellar wind velocity (right axis, dashed line) used as input for the simulations of the $1 M_{\odot}$ model during the AGB phase. The same is shown in Figure 2 for a star with an initial mass of $3.5 M_{\odot}$.

Finally, the interaction with the ISM is simulated by fixing the star at the center of the grid and allowing the ISM to flow into it at the outer boundary from $0^{\circ}$ to $90^{\circ}$. From $90^{\circ}$ to $180^{\circ}$ we set an outflow boundary condition. The temporal evolution of the stellar wind has been set within a small (five radial zones) spherical region centered on the symmetry axis, where reflecting boundary conditions are used. In doing that, we have assumed 
The Astrophysical Journal, 748:94 (16pp), 2012 April 1

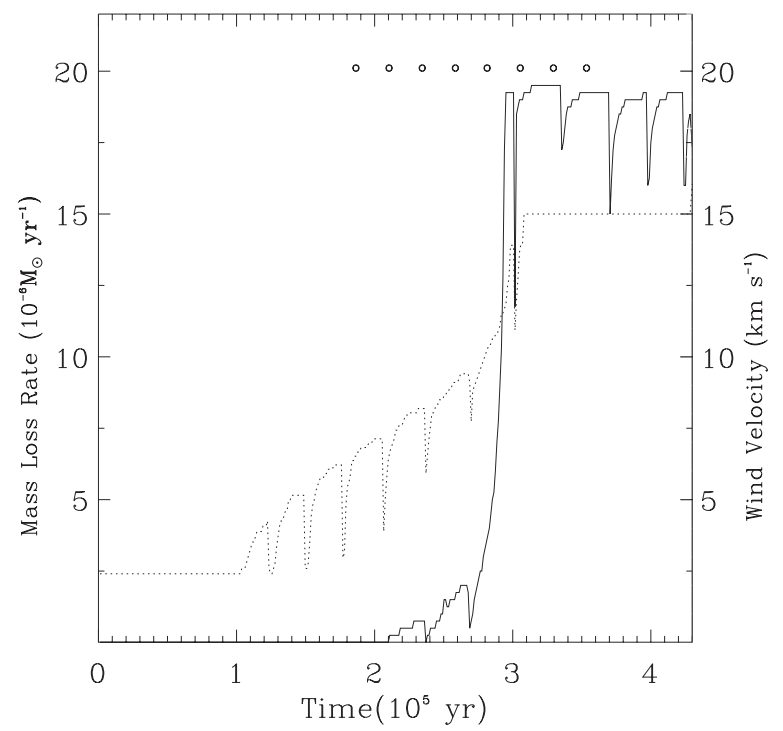

Figure 2. Same as Figure 1 but for a $3.5 M_{\odot}$ star. The circles in the top part of the plot mark the time at which the outputs in Figures 10 and 11 are shown.

that the ISM moves relative to the star perpendicular to the line of sight. Note that under the pure gas dynamics scheme with linear artificial viscosity used here, shock errors are expected to be small (Falle 2002).

\subsection{The Environment: The Physical Conditions of the ISM}

Regarding the gas component of the ISM and using the temperature as a discriminator, the diffuse ISM can be described within four major phases: the cold neutral medium (up to $100 \mathrm{~K}$ ), the warm neutral medium (with temperatures between $5000 \mathrm{~K}$ and $8000 \mathrm{~K})$, the warm ionized medium $(10,000 \mathrm{~K})$, and the hot ionized medium $\left(10^{6} \mathrm{~K}\right)$. The filling factors of each of the components are still controversial (Cox 2005). The values proposed originally by McKee \& Ostriker (1977) for the warm neutral (0.1), warm ionized (0.2), and hot ionized component $(0.7)$ have been revisited, mostly to account for a wide variety of observations that point toward a much lower filling factor for the hot component (less than $\sim 0.5$ ). The warm components are each thought to account for $\sim 0.2$ of the volume filling factor (see, e.g., Cox 2005). In short, most of the ISM volume relevant for this paper is occupied by hydrogen in its warm and ionized forms (see, e.g., Burton 1988; Kalberla \& Kerp 2009), and therefore, for the simulations, most of the ISM has been chosen to have the typical values of these two components. In this we have ignored the solid ISM component to model the physical conditions of the ISM. Although the treatment of dust is fundamental when considering matter-radiation interaction processes in the ISM, its dynamical effects are negligible given its small mass with respect to hydrogen $\left(\sim 3 \times 10^{-3}\right)$. We have not attempted to simulate the molecular gas component of the ISM given that it is found mostly in the form of discrete clouds occupying only a small fraction $(\sim 1 \%-2 \%)$ of the interstellar volume (see, e.g., Ferrière 2001).

The ISM densities used in this paper are within the range of values observed for the warm ISM component from 0.1 to $1 \mathrm{~cm}^{-3}$ (Kulkarni \& Heiles 1988). We have also explored typical values of the hot ISM component (with densities below $0.003 \mathrm{~cm}^{-3}$; Ferrière 2001). We have only considered the thermal component of the pressure to characterize the ISM, $P=n k T$ (where $k$ is the Boltzmann's constant). Although all
Villaver, Manchado, \& García-SEgura

Table 1

Model Parameters

\begin{tabular}{|c|c|c|c|c|c|c|}
\hline Run ID & $\begin{array}{c}v_{\mathrm{ISM}} \\
\left(\mathrm{km} \mathrm{s}^{-1}\right)\end{array}$ & $\begin{array}{c}n_{\mathrm{ISM}} \\
\left(\mathrm{cm}^{-3}\right)\end{array}$ & $\begin{array}{c}T_{\mathrm{ISM}} \\
(\mathrm{K})\end{array}$ & $\begin{array}{c}P_{\text {RAM }} \\
\left(\text { dyn } \mathrm{cm}^{-2}\right)\end{array}$ & Mach $_{\text {ISM }}$ & $\begin{array}{c}r_{\mathrm{so}} \\
\left(\times 10^{17} \mathrm{~cm}\right)\end{array}$ \\
\hline $\mathrm{R}_{1 \mathrm{M}} 10 \mathrm{~h}$ & 10 & 1 & 100 & $1.67 \times 10^{-12}$ & 8.5 & 0.77 \\
\hline $\mathrm{R}_{1 \mathrm{M}} 101$ & 10 & 0.1 & 100 & $1.67 \times 10^{-13}$ & 8.5 & 2.46 \\
\hline $\mathrm{R}_{1 \mathrm{M}} 20 \mathrm{~h}$ & 20 & 0.1 & 6000 & $6.69 \times 10^{-13}$ & 2.2 & 1.23 \\
\hline $\mathrm{R}_{1 \mathrm{M}} 201$ & 20 & 0.01 & 6000 & $6.69 \times 10^{-14}$ & 2.2 & 3.89 \\
\hline $\mathrm{R}_{1 \mathrm{M}} 301$ & 30 & 0.1 & 6000 & $1.33 \times 10^{-12}$ & 3.4 & 0.79 \\
\hline $\mathrm{R}_{1 \mathrm{M}} 50 \mathrm{~h}$ & 50 & 0.1 & 6000 & $4.18 \times 10^{-12}$ & 5.5 & 0.49 \\
\hline $\mathrm{R}_{1 \mathrm{M}} 501$ & 50 & 0.01 & 6000 & $4.18 \times 10^{-13}$ & 5.5 & 1.56 \\
\hline $\mathrm{R}_{1 \mathrm{M}} 851$ & 85 & 0.05 & 6000 & $6.04 \times 10^{-12}$ & 9.3 & 0.41 \\
\hline $\mathrm{R}_{1 \mathrm{M}} 100 \mathrm{~h}$ & 100 & 0.1 & 6000 & $1.67 \times 10^{-11}$ & 11.0 & 0.25 \\
\hline $\mathrm{R}_{1 \mathrm{M}} 1001$ & 100 & 0.01 & 6000 & $1.67 \times 10^{-12}$ & 11.0 & 0.79 \\
\hline $\mathrm{R}_{3.5 \mathrm{M}} 20 \mathrm{~h}$ & 20 & 0.1 & 6000 & $6.69 \times 10^{-13}$ & 2.2 & 1.34 \\
\hline $\mathrm{R}_{3.5 \mathrm{M}} 50 \mathrm{~h}$ & 50 & 0.1 & 6000 & $4.18 \times 10^{-12}$ & 5.5 & 0.54 \\
\hline $\mathrm{R}_{3.5 \mathrm{M}} 501$ & 50 & 0.01 & 6000 & $4.18 \times 10^{-13}$ & 5.5 & 1.72 \\
\hline
\end{tabular}

the phases of the ISM are thought to coexist in roughly thermal pressure equilibrium, it has been shown that the average thermal component is less than one-third of the total pressure in the midplane and that the non-thermal pressure components (cosmic rays and magnetic fields) each take roughly the other two-thirds (Ferrière 2001). Typical mid-plane values of thermal pressure are of the order of $\sim 10^{-12}$ dyn $\mathrm{cm}^{-2}$, decreasing outward (Cox 2005).

The values used in our simulations range between $10^{-15}$ and $1.38 \times 10^{-13} \mathrm{dyn} \mathrm{cm}^{-2}$ (with most of them $8 \times 10^{-14} \mathrm{dyn} \mathrm{cm}^{-2}$ ), therefore representing typical values off the mid-plane (see Table 1). We have not included magnetic fields or turbulent motions in the simulations.

\subsection{The Relative Velocity: Stellar Dynamics}

To model the interaction process, it is important to characterize dynamically the three major stellar components of the Galaxy: the thin disk, the bulge, and the halo. Population I stars belong to the disk and follow a differential rotation curve around the center in nearly circular orbits with angular rotation rates a decreasing function of their radial distance. Disk stars have a velocity dispersion 10-40 $\mathrm{km} \mathrm{s}^{-1}$ (MacDonald \& Bailey 1981), which causes them to execute small oscillations about a perfectly circular orbit, both in the Galactic plane (epicycles) and in the vertical direction. The thin disk has a radius $\approx 25-30 \mathrm{kpc}$ and effective thickness $\approx 400-600$ pc. We have characterized the population of stars belonging to the disk by using relative velocities in the $10-50 \mathrm{~km} \mathrm{~s}^{-1}$ range (see Table 1). Higher ISM densities have been used for the lower velocity models in order to be consistent with the conditions found by stars moving closer to the mid-plane. For the same reason, we have decreased the ISM density when simulating stars moving with larger velocities away from the Galactic plane. Overall, the ISM density range used spans two orders of magnitude (from 1 to $0.01 \mathrm{~cm}^{-3}$ ).

How to characterize dynamically the stellar population of the halo is not so straightforward. The halo extends out to more than $30 \mathrm{kpc}$ from the center (Binney \& Merrifield 1981), and the orbital behavior of the halo (Population II) stars is still not clear, but it seems to contain a combination of stars with extreme retrograde orbits (Carney et al. 1997), highly inclined orbits, and stars that do not move in regular orbits at all (Eggen et al. 1962). In addition, there are vertical and radial kinematic gradients (see, e.g., Majewski 1993; Beers et al. 2000; Chiba \& Beers 2000; 

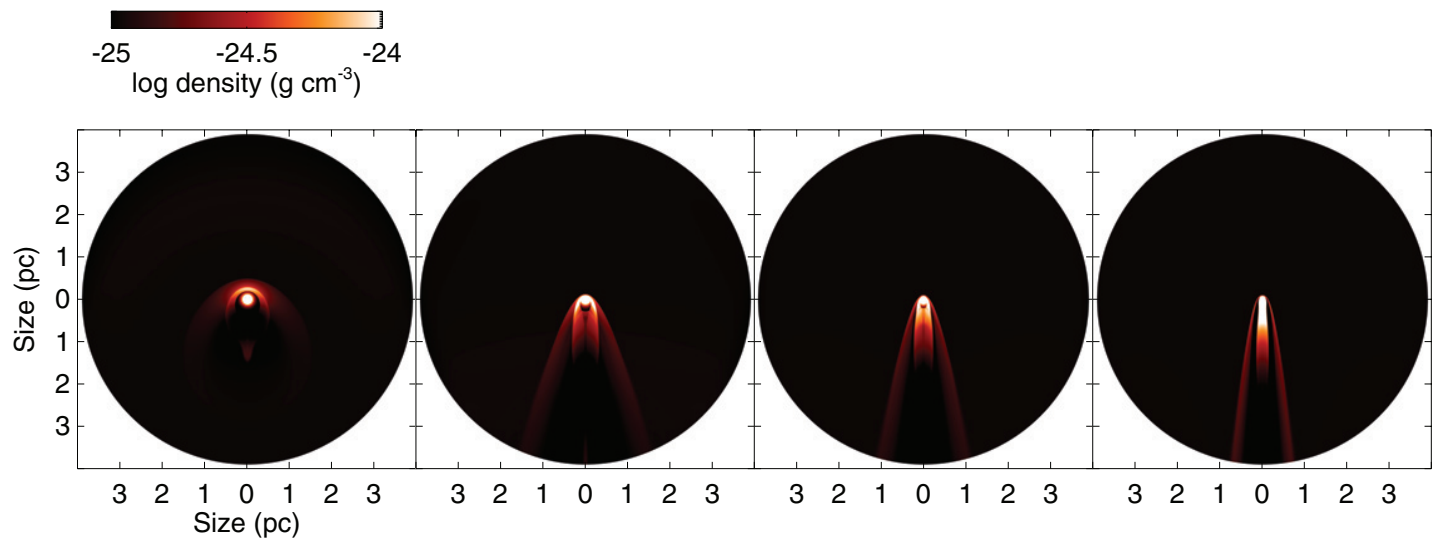

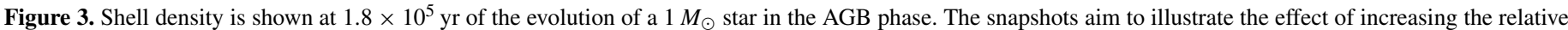

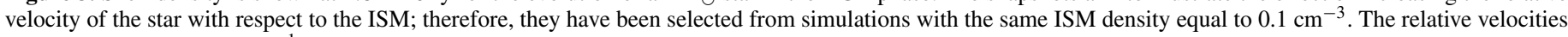
are $10,30,50$, and $100 \mathrm{~km} \mathrm{~s}^{-1}$, increasing from left to right.

(A color version of this figure is available in the online journal.)

Helmi 2008). To represent this population, we have performed simulations with larger relative velocities $\left(85-100 \mathrm{~km} \mathrm{~s}^{-1}\right)$.

We have not attempted to model the conditions relevant to the galactic bulge, although any of the simulations described above can be applied to the lower end of the velocity range for AGB stars.

A summary of the parameters used for the simulations is given in Table 1. Column 1 is the run ID name where the first number underscored refers to the stellar mass; Column 2 lists the velocity of the star relative to the ISM; Columns 3 and 4 give the value of the adopted ISM density and temperature, respectively; Column 5 lists the value of the ram pressure for the simulation and Column 6 the Mach number of the ISM; finally, Column 7 is the stand-off distance (see Section 3).

\section{RESULTS: EARLY-AGB CIRCUMSTELLAR SHELLS}

\subsection{The Early-AGB Phase: Constant Wind}

During the early-AGB phase, the evolution is characterized by a constant free-streaming stellar wind with velocity $v_{* w}$ and mass-loss rate $\dot{M}_{* w}$. For a star moving supersonically with a velocity $v_{\text {ISM }}$ through an ISM with density $n_{\text {ISM }}$, the distance from the star at which the ram pressure of the free-streaming wind equals that of the ISM is given by (see, e.g., van Buren et al. 1990; Mac Low et al. 1991)

$$
\begin{aligned}
r_{\mathrm{so}}= & 5.5 \times 10^{17}\left(\frac{\dot{M}_{* w}}{10^{-8}}\right)^{1 / 2}\left(\frac{v_{* w}}{10^{5}}\right)^{1 / 2} \\
& \times \mu^{-1 / 2} n_{\mathrm{ISM}}^{-1 / 2}\left(\frac{v_{\mathrm{ISM}}}{10^{5}}\right)^{-1}
\end{aligned}
$$

where $\mu$ is the dimensionless mean molecular weight, $r_{\text {so }}$ is given in $\mathrm{cm}, \dot{M}_{* w}$ in $M_{\odot} \mathrm{yr}^{-1}$, and the densities and velocities are given in $\mathrm{cm}^{-3}$ and $\mathrm{cm} \mathrm{s}^{-1}$, respectively.

Equation (1) is applicable as long as the stellar wind is kept constant at the inner boundary, or the wind is constant long enough to reach pressure equilibrium with the ISM. For AGB stars that is the case only during the early-AGB evolution (see Figures 1 and 2). The validity of this early stationary approach to calculate the analytical stand-off distance is broken relatively early in the AGB evolution: at $\sim 1.8 \times 10^{5} \mathrm{yr}$ into the evolution of the $1 M_{\odot}$ star, and at $\sim 1 \times 10^{5} \mathrm{yr}$ for the $3.5 M_{\odot}$ stellar model. The values $r_{\text {so }}$ calculated from Equation (1) using the
early-AGB wind $\left(0.1 \times 10^{-7} M_{\odot}\right.$ and $2-2.5 \mathrm{~km} \mathrm{~s}^{-1}$ winds $)$ are given in Column 7 of Table 1 .

At this early stage, the structure of the circumstellar shell in the simulations is relatively simple, showing the characteristic bow-shock structure in the direction of the movement. Figure 3 shows the result in the density structure, in logarithm scale, of the interaction for relative velocities of $10,30,50$, and $100 \mathrm{~km} \mathrm{~s}^{-1}$ (from left to right). The panels have been taken at the end of the stationary wind phase (at $\sim 1.8 \times 10^{5} \mathrm{yr}$ ) of the evolution of a $1 M_{\odot}$ star through an ISM with a density of $0.1 \mathrm{~cm}^{-3}$.

The simulations show an asymmetric shell structure that develops early in the evolution of the shell. Its morphology is highly influenced by the relative velocity of the interaction (see Figure 3). The most straightforward effect of increasing the velocity of the interaction with the ISM is the closing of the opening angle of the bow shock. It is important to note as well that the size of the cometary tail in the downward direction increases with the relative velocity of the star with respect to the ISM. The value of $r_{\mathrm{so}}$, obtained from Equation (1) and given in Table 1, agrees well with the sizes obtained from the simulations. Note that comparing the analytical and the numerical values is only meaningful for those models in which the shell, at this stage, is larger than the grid resolution in the radial direction $(0.01 \mathrm{pc})$.

It is important to note here that Wareing et al. (2006, 2007a, 2007c), in their study of the interaction with the ISM, only consider a single wind (with a constant mass loss of either 1 , 5,10 , or $50 \times 10^{-7} M_{\odot} \mathrm{yr}^{-1}$ depending on the velocity used for the interaction) for the whole AGB evolution. A single AGB wind has been used as well by Raga et al. (2008) and Esquivel et al. (2010). The only time in which the AGB wind is constant in our simulations is during the early-AGB phase described above; therefore, this is the only time at which it is meaningful to compare the results of our simulations with the ones of the literature. A more detailed comparison with the numerical simulations in the literature is given in the discussion section.

\section{RESULTS: THE EVOLUTION OF THE CSE AS THE STAR EVOLVES ALONG THE AGB PHASE}

\subsection{Low-velocity Models: $v=10$ and $20 \mathrm{~km} \mathrm{~s}^{-1}$}

Figure 4 illustrates the structure of the CSE of a moving star as it evolves in the H-R diagram along the AGB phase. Figure 4 corresponds to the first model listed in Table 1 , model $\mathrm{R}_{1 \mathrm{M}} 10 \mathrm{~h}$, 

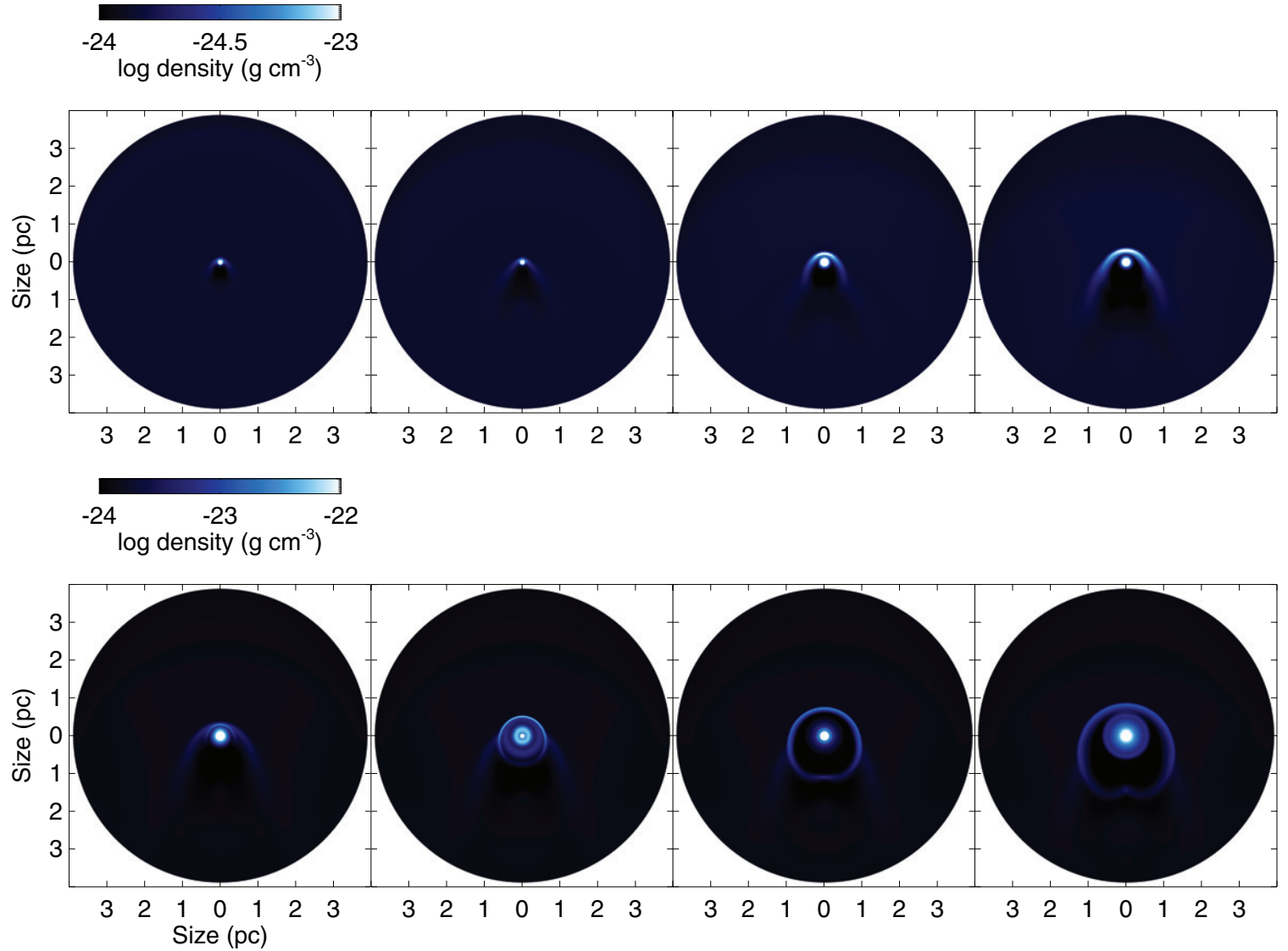

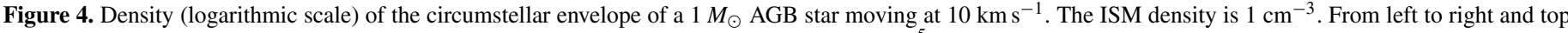

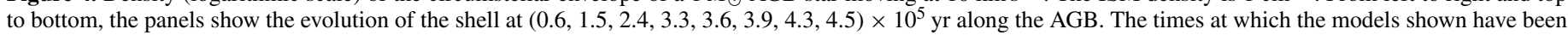
selected are marked in Figure 1 where we plot the stellar wind used in the simulations.

(An animation and a color version of this figure are available in the online journal.)

and aims to be representative of a Population I star on the lower end of the expected velocity dispersion, $10 \mathrm{~km} \mathrm{~s}^{-1}$ and evolving close to the Galactic plane.

In Figure 4 from left to right and top to bottom, the panels show the evolution of the shell at $(0.6,1.5,2.4,3.3,3.6,3.9,4.3$, $4.5) \times 10^{5} \mathrm{yr}$ along the AGB. In the first two panels of Figure 4 the density structure is shown at a time when the stellar wind has still a very small momentum $\left(10^{-8} M_{\odot} \mathrm{yr}^{-1}, 2 \mathrm{~km} \mathrm{~s}^{-1}\right)$. Yet the characteristic feature of the interaction has already developed: a bow shock in the direction of the movement, that is, toward the top of the page.

As the star evolves (see Figure 1), the mass-loss rate (and wind velocity) increases and the CSE begins to grow in the leading direction of the movement (third and fourth top panels of Figure 4), given that the stellar wind has enough momentum to compete with the ram pressure provided by the ISM. In all, the stellar wind is always allowed to expand faster along the opposite direction of the movement given that the ambient pressure is smaller and the opening angle of the bow shock is maintained. It is important to note how different the shape of the CSE is along the evolution of the star on the AGB. This is the result of the wind continuously changing in the inner boundary of the grid.

In the first panel on the bottom left, the star is undergoing a second mass-loss increase, which is orders of magnitude larger than any previous mass loss. The stellar wind propagates through the density profile created by the previously ejected material, and a shock region develops. This shock is not with the ISM material but between subsequent episodes of mass loss. No shell is present in the opposite direction of the movement where the wind expands subsonically and no discontinuity can develop. It is only toward the end of the AGB phase that the wind is dense enough to create a shell in the direction opposite to the movement.

Note that, in general, the stellar wind encounters a lower pressure opposite to the movement and is able to expand further in that direction. As a consequence, the star is displaced from the geometrical center of the shell and an outer asymmetric CSE is formed.

The ISM density used in this model, $1 \mathrm{~cm}^{-3}$, aims to represent the average conditions of the cold neutral medium in the Galactic plane, and accordingly the temperature of the ISM gas was set at $100 \mathrm{~K}$. The large Mach number of the ISM in this case results in a larger shock compression given that the shell is cooling radiatively.

It is expected that a large number of AGB stars might be moving at such low velocities relative to the local ISM. A large ISM density, even though associated with a very slow moving star, can generate an asymmetric shell. The stellar wind gets deflected by the high ram pressure of the ISM and expands more easily in the direction opposite to the interaction. The stellar wind always expands inside the cavity cleared by the previous stellar activity, not showing any sign of the interaction process. The asymmetry of the interaction is only observable in the outermost shell. For the parameters used in this model a large asymmetric outer shell is formed that is a factor of two larger in 

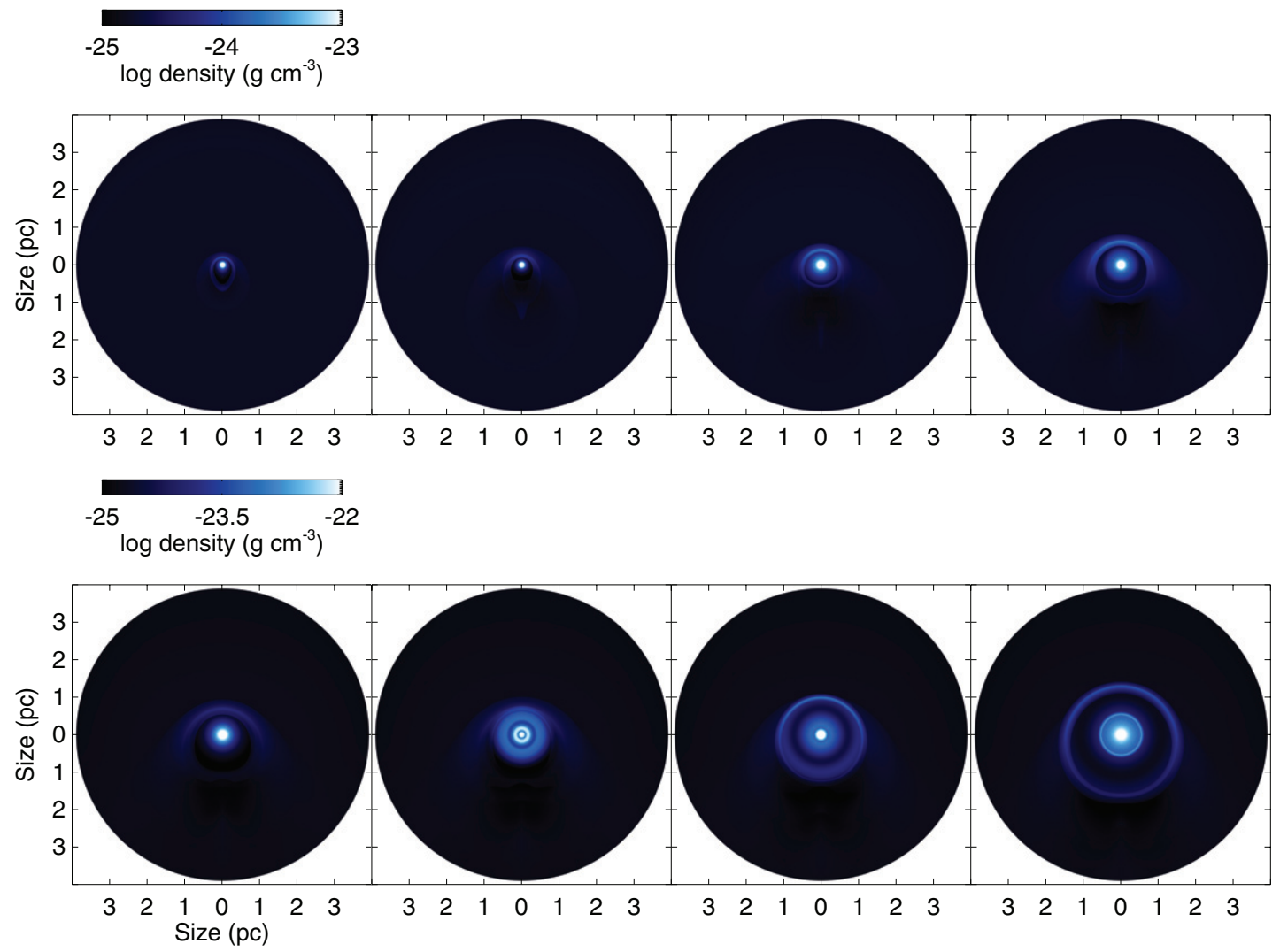

Figure 5. Same as Figure 4 but for an ISM with density $0.1 \mathrm{~cm}^{-3}$.

(An animation and a color version of this figure are available in the online journal.)

the downstream direction compared to the upstream direction. No morphological feature of the interaction is recorded in the inner CSE at the end of the AGB given that the wind is expanding almost unperturbed within the asymmetric outer cavity created by the interaction. No tail in the opposite direction of the movement remains in this model. The bulk of the mass lost by the star is left behind along the movement; only the latest $10,000 \mathrm{yr}$ of mass loss are recorded in the shell structure.

Figure 5, model $\mathrm{R}_{1 \mathrm{M}}$ 101, shows the CSE density structure formed by the interaction of a $1 M_{\odot}$ AGB star with an ISM, with a density 10 times lower than the one shown in Figure 4. The snapshots in the figures have always been chosen at the same time in the evolution, unless noted otherwise, in order to better show the effects of changing the conditions of the environment.

The main morphological features of the interaction described previously are also present here, and, as in the previous case, the mass lost by the star is continuously deflected in the downstream direction of the movement. The important difference in this case is that a smaller ISM density allows for the formation of a shell in the downstream direction and the lower ram pressure of the ISM allows a larger growth of the shell in the upstream direction. As a consequence, the asymmetry generated by the interaction on the outermost shell is less obvious in this case and translates to a small displacement of the location of the central star with respect to the geometrical center of the envelope once the massloss rate associated with the last thermal pulses takes place (last two bottom panels). In general, once the mass loss reaches its highest rate at the end of the AGB, the effect of the interaction on the shell is very small.

Models evolving at relative velocities of $20 \mathrm{~km} \mathrm{~s}^{-1}$ at densities of 0.1 and $0.01 \mathrm{~cm}^{-3}$ were presented in Villaver et al. (2003).
Note that in model $\mathrm{R}_{1 \mathrm{M}} 201$ we have used an ISM density of $0.01 \mathrm{~cm}^{-3}$, resulting in the lowest ram pressure considered in this work. These models show the same main features of the models described above. The AGB wind forms a bow shock upstream of the central star. The mass lost by the star as it ascends the AGB does not interact directly with the ISM; it expands within the bow shock and gets deflected downstream. It is only the mass lost at the end of the AGB phase that generates enough pressure to reach the bow shock. This mass loss also leads to the growth of the bow-shock structure in the direction of the movement and generates shocks with the stellar mass loss within this shell. The asymmetry is still noticeable at the end of the AGB; the bulk of the mass lost by the star is deflected in the downward direction. Besides losing the spherical symmetry, the CSE formed under a low-velocity interaction has a smaller size and contains a lower mass than when formed under zero relative velocity (Villaver et al. 2002a). This statement is true also for the models moving at $10 \mathrm{~km} \mathrm{~s}^{-1}$.

\subsection{Intermediate-velocity Models: 30 and $50 \mathrm{~km} \mathrm{~s}^{-1}$}

Figures 6 and 7 show simulations of a low-mass star moving with intermediate relative velocities, 30 and $50 \mathrm{~km} \mathrm{~s}^{-1}$, through an ISM with a density of $0.1 \mathrm{~cm}^{-3}$. These models aim to match the velocity dispersion of stars belonging to the disk population. As with the figures shown in the previous subsection, the sequence of outputs have been selected at the same times as shown in Figure 5 and marked in the top of Figure 1.

As for the low-velocity models shown before, in the first two top panels the AGB wind still has the characteristics of the earlyAGB phase, and a bow shock forms in the leading direction. The stationary wind phase lasts long enough for the AGB wind to 

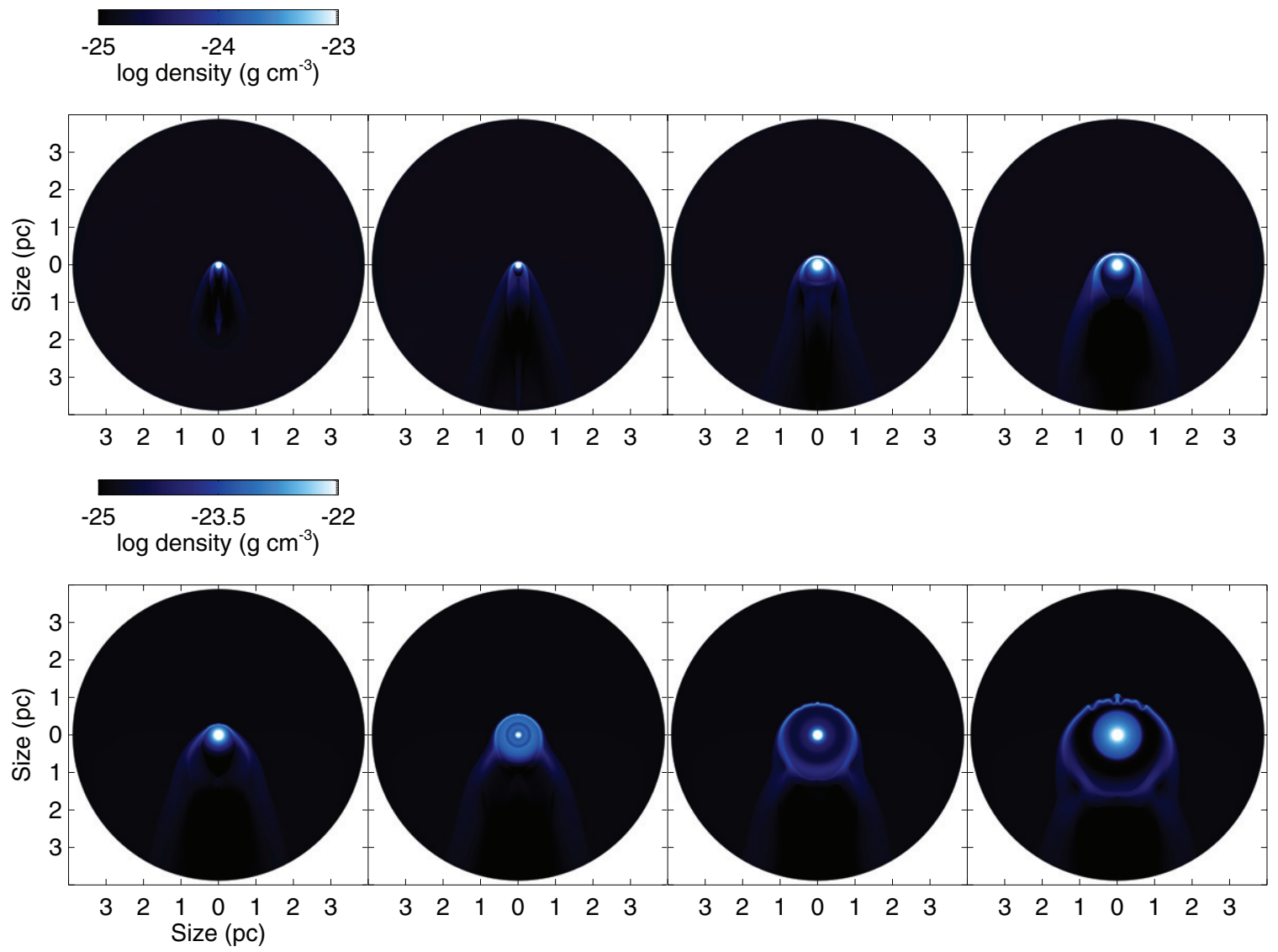

Figure 6. Same as Figure 4 but for a star moving at $30 \mathrm{~km} \mathrm{~s}^{-1}$ through an ISM with density $0.1 \mathrm{~cm}^{-3}$.

(An animation and a color version of this figure are available in the online journal.)

reach pressure equilibrium with the ISM. Thus, the bow shock reaches a stable position ahead of the star. Both the opening angle of the bow shock and the distance from the star decrease as the velocity that characterizes the ISM interaction increases.

The main difference between the models with low and intermediate velocities can be seen already in the third and fourth top panels of Figures 6 and 7 shown at $2.5 \times 10^{5}$ and $3.4 \times$ $10^{5} \mathrm{yr}$ into the AGB evolution. For these intermediate relative velocities the previous mass loss gets deflected behind the star more efficiently and thus the stellar wind encounters the ISM directly. The main characteristic of the interaction for these models is the development of instabilities in the bow shock. When the wind expands throughout surroundings already cleared by the previous stellar activity, no instabilities are developed. That is the case for the models with velocities of 10 and $20 \mathrm{~km} \mathrm{~s}^{-1}$. However, as the velocity of the interaction increases, the outermost shell is subject to instabilities as predicted by Blondin \& Koerwer (1998) (see Section 6).

The wind expands faster along the hot cavity left behind by the star. The formation of a highly asymmetric shell that grows in size in the perpendicular and in the opposite directions of the movement can be seen in the shell evolution in Figures 6 and 7. Again the leading part of the CSE expands away from the star only when the stellar wind has enough momentum (either the wind velocity increases or the mass-loss rate) to compete with the ISM ram pressure. And, in the few instances that the ISM pressure is larger than the one provided by the shell in the leading direction, the ISM pushes the shell inward, creating the seeds for the formation of an unstable flow. The formation of instabilities highly influences the CSE shell structures as the unshocked ISM is able to penetrate deep into the shell as it breaks up.

In the downstream direction the wind expands within the tunnel left behind by the star and an elongated tail grows. Mass is constantly flowing away from the head of the bow shock, feeding the cometary structure. The tail material is formed by a mixture of material stripped from the head of the bow shock (and cooling as it flows downstream around the bow shock toward the back of the star) and wind material directly ejected by the star in the downstream direction.

Decreasing the density of the ISM for the same velocity has an enormous influence in the shell evolution as shown in model $\mathrm{R}_{1 \mathrm{M}}$ 501. In fact, this model has a ram pressure comparable with the first model listed in Table 1 for a velocity of $20 \mathrm{~km} \mathrm{~s}^{-1}$ and ISM density of $0.1 \mathrm{~cm}^{-3}$ (Villaver et al. 2003). Both models show that the effects of the interaction in the morphology of the outer shell are small toward the end of the AGB evolution. However, the tail in the opposite direction of the movement is still formed as ram pressure is stripping matter from the shell. Evolution through low ram pressure environments $\left(\approx 10^{-13} \mathrm{dyn} \mathrm{cm}^{-2}\right)$ does not produce a strong morphological feature at the tip of the AGB. However, no matter how small the ram pressure of the interaction is, the morphology is strongly affected as the star ascends the AGB. Still, this effect is important in the evolution of the CSE shells. Ram pressure stripping constantly removes the mass of the CSE, and as a consequence lower density shells are formed. The external pressure provided by the external medium reduces the expansion velocity of the 

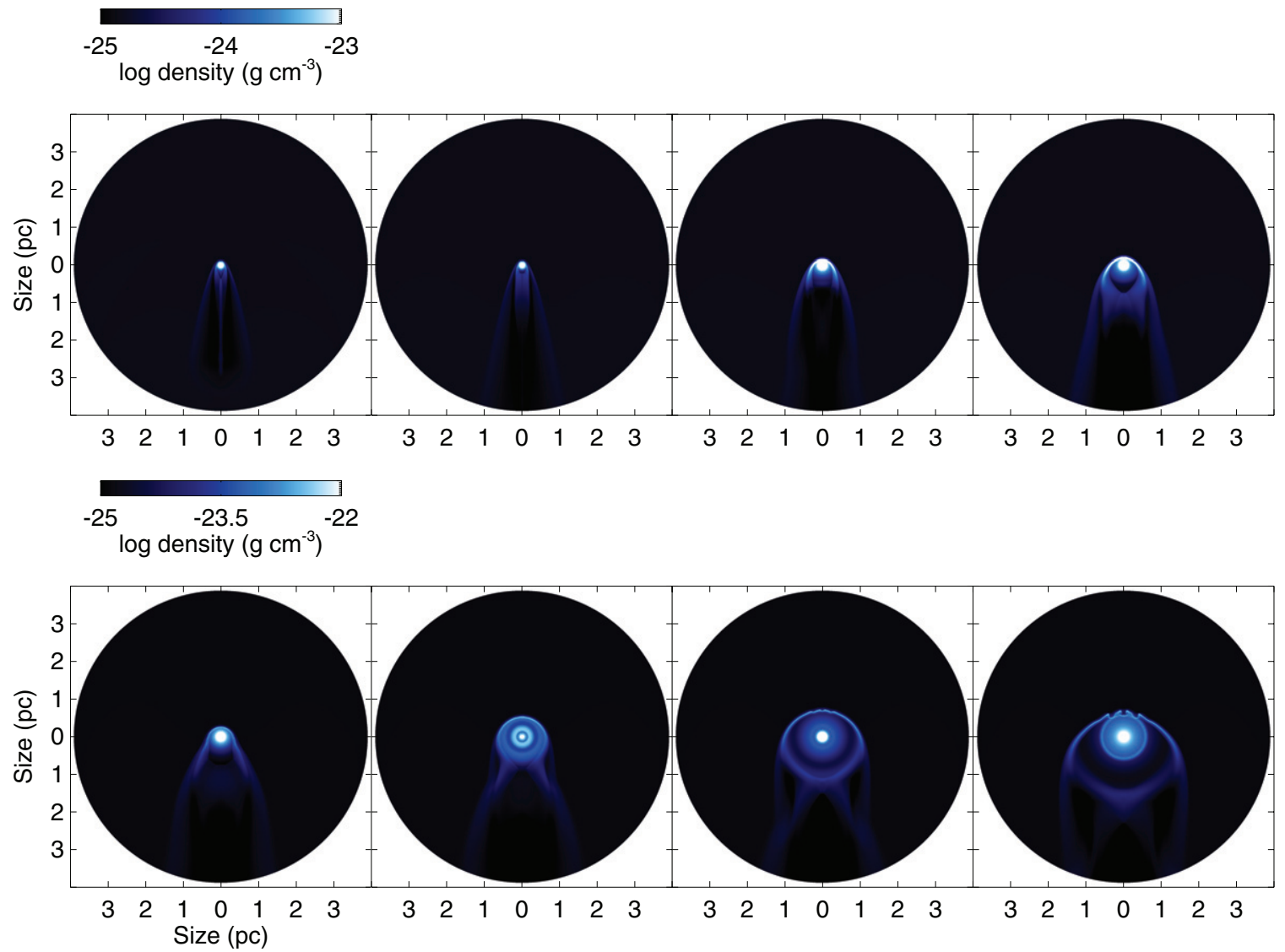

Figure 7. Same as Figure 4 but for a star moving at $50 \mathrm{~km} \mathrm{~s}^{-1}$ through an ISM with density $0.1 \mathrm{~cm}^{-3}$.

(An animation and a color version of this figure are available in the online journal.)

shell in the direction of the interaction, increasing it along the opposite one.

\subsection{High-velocity Models: 85 and $100 \mathrm{~km} \mathrm{~s}^{-1}$}

A relative velocity of $100 \mathrm{~km} \mathrm{~s}^{-1}$ for the ISM interaction is shown in Figure 8, model $\mathrm{R}_{1 \mathrm{M}} 100 \mathrm{~h}$. A narrow, confined bow shock is formed in the upstream direction, and a long tail is prominent downstream of the motion. This model has the largest ram pressure of all the models computed and shows the strongest features of the interaction. At the time when the first increase in mass loss takes place (third top panel from the left), the flow is already dynamically unstable, and soon the bow shock is broken by the instabilities (see fourth top panel). The tail formed in the downstream direction is Kelvin-Helmholz unstable. In the tail, wind material expanding outward from the star interacts with material generated in the eddies of the outer shell, material that is moving inward toward the star. A turbulent region develops where they encounter each other, and several shocks are formed within the tail.

The stellar wind interacts directly with the ISM; it becomes unstable, breaks, and gets deflected. The ISM can penetrate in the leading direction close to the star when the wind has its minimum mass loss between superwind (SW) events. At the time of the last increase in the stellar mass loss, the shell in the leading direction grows in size despite being highly unstable. At the end of the AGB, the CSE shows a largely turbulent morphology with several condensations caused by the shell fragmentation. The overall morphology of the bow shock is maintained by tracing the tips of the condensations caused by the instabilities. A similar unstable flow has been shown to develop in model $\mathrm{R}_{1 \mathrm{M}} 851\left(v=85 \mathrm{~km} \mathrm{~s}^{-1}\right.$ and $n=0.05 \mathrm{~cm}^{-3}$; Szentgyorgyi et al. 2003).

Figure 10 shows the same as Figure 8 but reducing by a factor of 10 the density of the ISM, which can be considered more realistic given the high velocity considered. A lower density of the interaction reduces the ram pressure and also allows us to study the effect of the ISM density in the interaction. In this model, $\mathrm{R}_{1 \mathrm{M}} 1001$, the flow is dynamically unstable as well. However, given the lower ram pressure, the ISM cannot penetrate close to the star when the stellar wind reaches its minimum. So the mixing between ISM and wind material is not so efficient. The tail in the downstream direction is very prominent in this model given that the ISM density is lower and therefore any density enhancement has more contrast. The material gets deflected downstream and mixed efficiently on the tail of the interaction, while in the direction of the movement the shell, although unstable, does not break up completely and in fact is allowed to grow in the direction of the movement.

Note that this model $\mathrm{R}_{1 \mathrm{M}} 1001\left(v=100 \mathrm{~km} \mathrm{~s}^{-1}\right.$ and $n=0.01$ $\left.\mathrm{cm}^{-3}\right)$ has the same ISM ram pressure as model $\mathrm{R}_{1 \mathrm{M}} 10 \mathrm{~h}(v=$ $10 \mathrm{~km} \mathrm{~s}^{-1}$ and $n=1 \mathrm{~cm}^{-3}$ ). However, in order to adopt realistic ISM conditions, the temperature of the ISM had to be modified according to the ISM density. As a result, the Mach number of the ISM is very different in these three cases (see Table 1). Despite the fact that these models have the same stellar input and are evolving against the same ram pressure of the external medium, the CSEs formed are radically different. The $\mathrm{R}_{1 \mathrm{M}} 10 \mathrm{~h}$ model does not develop instabilities, the outer shell does not break up, and a cometary tail does not form either. This shows that ram pressure is not the main parameter in order to determine 

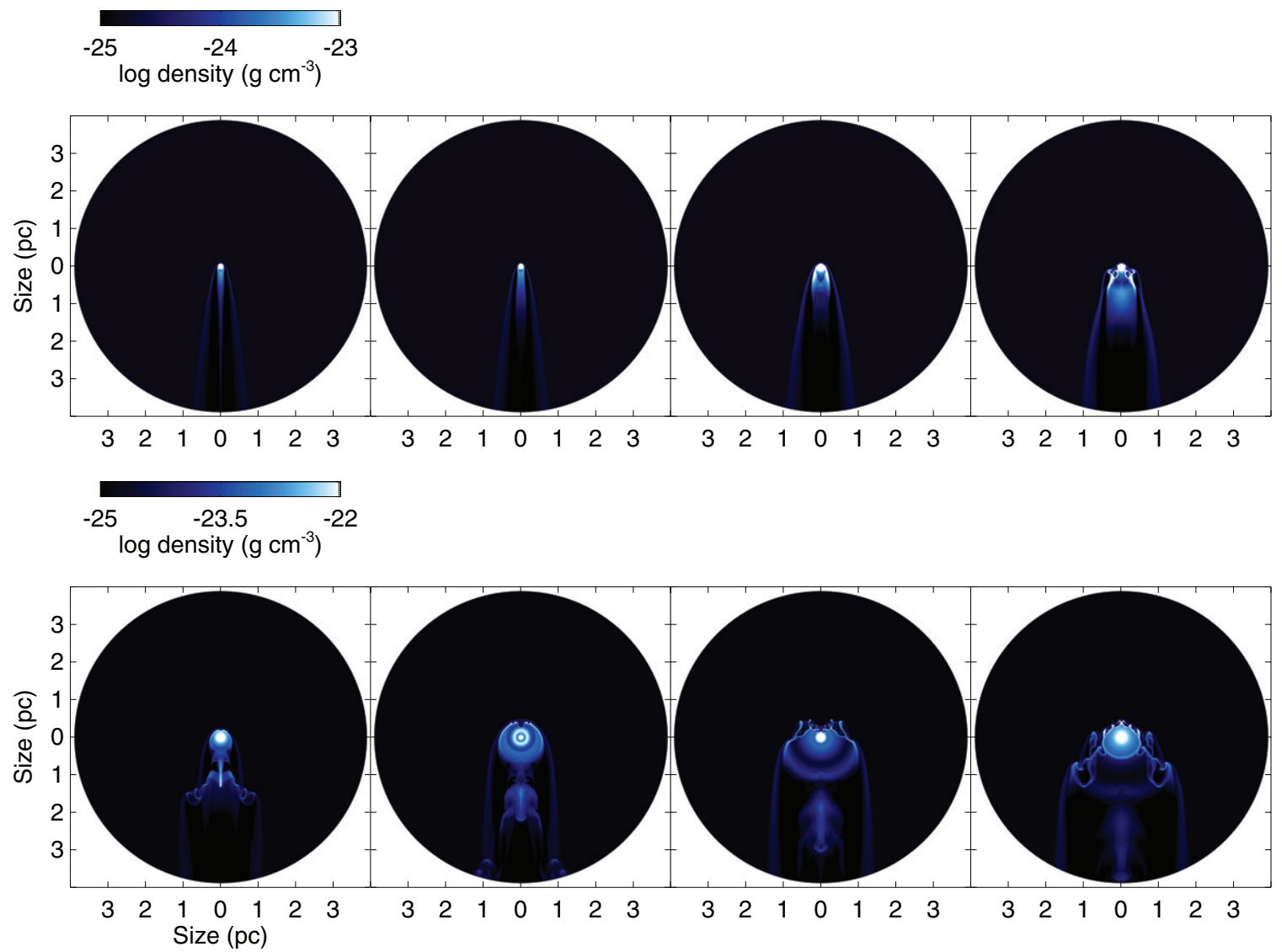

Figure 8. Same as Figure 5 but for a star moving at $100 \mathrm{~km} \mathrm{~s}^{-1}$ through an ISM with density $0.1 \mathrm{~cm}^{-3}$.

(An animation and a color version of this figure are available in the online journal.)

the shell morphology. Ram pressure, however, determines the sizes of the outermost shell at the end of the AGB evolution, and it can be seen that the shell sizes are the same for the two models (when tracing the tips of the condensations caused by the instabilities).

In Figure 9 we show the same as in Figure 3, but with the outputs taken later on in the evolution. All the models evolved through the same ISM density of $0.1 \mathrm{~cm}^{-3}$, and the relative velocities, from left to right, are $10,30,50$, and $100 \mathrm{~km} \mathrm{~s}^{-1}$. Before, we pointed out that the most straightforward effect of increasing the velocity of the interaction with the ISM is the closing of the opening angle of the bow shock. Here, we need to emphasize the more prominent effect of the instabilities in the shell morphology as the relative velocity of the interaction increases. It is important to note as well that the cometary tail in the downward direction becomes more prominent as the relative velocity of the star with respect to the ISM increases.

\section{MIRA AS A TEST OF A HIGH-VELOCITY INTERACTION}

Mira is a thermally pulsating AGB star that is expected to experience wind modulations associated with its thermal pulse cycle. $\mathrm{CO}$ observations of the system trace the current and/or most recent mass loss to be $1.7 \approx \times 10^{-7} M_{\odot} \mathrm{yr}^{-1}$ (Ryde \& Schöier 2001). Mira is moving at $\approx 125 \mathrm{~km} \mathrm{~s}^{-1}$ through its surrounding medium. Located at 107 pc (Knapp et al. 2003), the physical sizes of its far-ultraviolet cometary tail and bowshock head are 4 and $0.1 \mathrm{pc}$, respectively. The tail has also been detected in HI out to $0.4 \mathrm{pc}$ from the star (Matthews et al.
2008). Herschel's PACS and Spitzer observations reveal that the arcs seen around Mira's head likely result from a combination of the projected three-dimensional structures resulting from the interaction of Mira's wind with its companion on the one hand and with the ISM on the other hand (Ueta 2008; Mayer et al. 2011).

Although we have not run any specific model to match the observations of Mira (Martin et al. 2007), we have a number of simulations with a range of parameters similar to those used in the literature specifically devoted to that matter (Wareing et al. 2007c; Esquivel et al. 2010) that allow us to perform a limited qualitative comparison among models. We are excluding from the comparison (1) the models of Raga et al. (2008), since they focused on reproducing the complex double bow shock in the cometary head using a latitude-dependent wind associated with the binary component, and (2) the models of Raga \& Cantó (2008), given their analytical nature.

In Table 2 we have summarized the relevant parameters to allow the comparison: Column 1 gives the relevant reference (where models $\mathrm{R}_{1 \mathrm{M}} 1001$ and $\mathrm{R}_{1 \mathrm{M}} 1001$ allude to this work); Column 2 gives the relative velocity of the interaction; Columns 3 and 4 give the density and temperature adopted for the ISM; finally, Columns 5-7 provide the stellar wind parameters adopted in the simulations in terms of mass loss, wind velocity, and temperature, respectively.

Note that in our models, the wind is always changing in the inner boundary according to what is expected from a thermally pulsating AGB star; however, until $\approx 1.8 \times 10^{5} \mathrm{yr}$ into the evolution the wind is constant, and until $\approx 2.8 \times 10^{5} \mathrm{yr}$, although variable in mass loss, wind velocity and temperature still have 

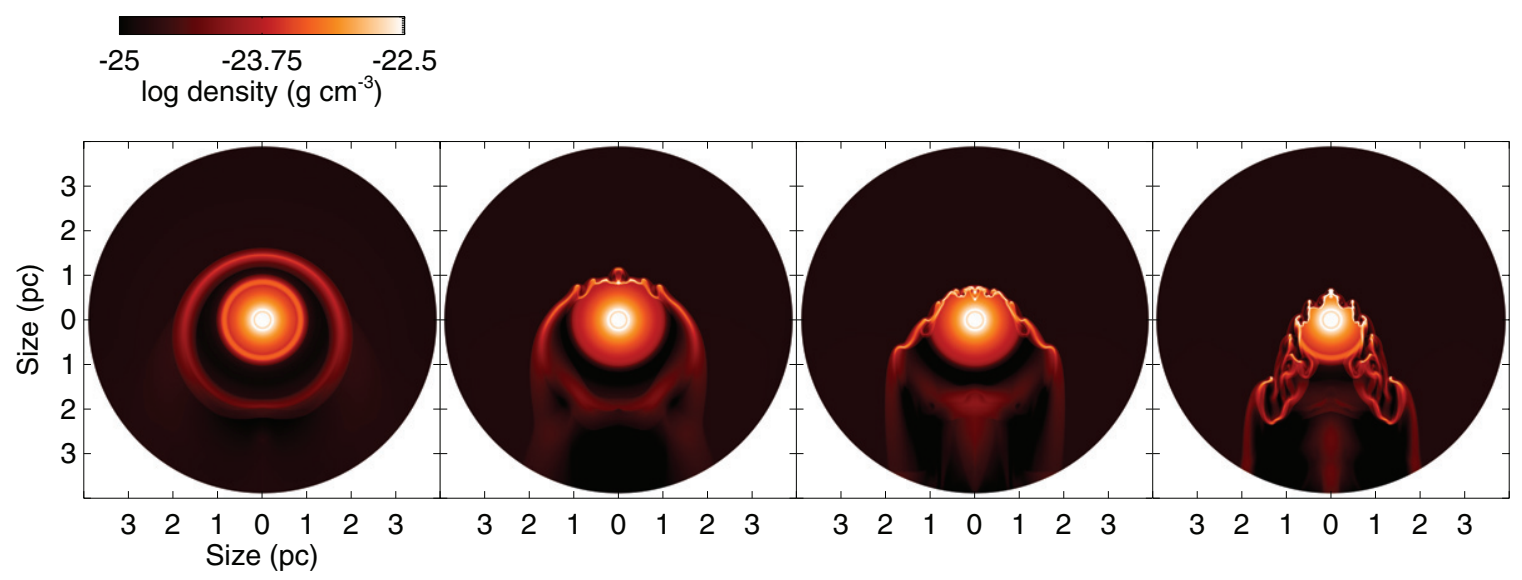

Figure 9. Snapshots taken at $4.74 \times 10^{5} \mathrm{yr}$ of a $1 M_{\odot}$ star moving (from left to right) at $10,30,50$, and $100 \mathrm{~km} \mathrm{~s}^{-1}$.

(A color version of this figure is available in the online journal.)

Table 2

Comparison between Models under the Parameters for Mira

\begin{tabular}{|c|c|c|c|c|c|c|}
\hline Reference & $\begin{array}{c}v_{\text {ISM }} \\
\left(\mathrm{km} \mathrm{s}^{-1}\right)\end{array}$ & $\begin{array}{c}n_{\mathrm{ISM}} \\
\left(\mathrm{cm}^{-3}\right)\end{array}$ & $\begin{array}{c}T_{\text {ISM }} \\
(\mathrm{K})\end{array}$ & $\begin{array}{c}\dot{M}_{\text {wind }} \\
\left(10^{-7} M_{\odot} \mathrm{yr}^{-1}\right)\end{array}$ & $\begin{array}{c}v_{\text {wind }} \\
\left(\mathrm{km} \mathrm{s}^{-1}\right)\end{array}$ & $\begin{array}{c}T_{\text {wind }} \\
(\mathrm{K})\end{array}$ \\
\hline Model $\mathrm{R}_{1 \mathrm{M}} 1001$ & 100 & 0.01 & 6000 & $0.1-1.4$ & $2-7$ & 2700 \\
\hline Model $\mathrm{R}_{1 \mathrm{M}} 100 \mathrm{~h}$ & & 0.1 & & & & \\
\hline Wareing et al. (2007b) & 130 & 0.03 & 8000 & $1-3$ & 5 & 10,000 \\
\hline Esquivel et al. (2010) & 125 & $\begin{array}{c}1 \\
0.05\end{array}$ & $\begin{array}{c}1000 \\
10^{6}\end{array}$ & 3 & 5 & 100 \\
\hline
\end{tabular}

parameters similar to those used in other models to compare with the observations of Mira. The outputs of the simulations that are relevant to this discussion are those shown in the top four panels of Figures 8 and 10. Note that only the first two are computed under a constant wind assumption.

As in previous models, the location of the stand-off distance and the size of the tail are similar to those observed once we take into account the slight differences in both the wind parameters and the ISM conditions for the interaction (see the first panel in the top left of Figures 8 and 10). In both of our simulations, under a low $\left(0.01 \mathrm{~cm}^{-3}\right)$ and high $\left(0.1 \mathrm{~cm}^{-3}\right)$ density environment we obtain narrow cometary tails as a consequence of the interaction. Note that although the model evolving in a low-density environment is similar to that computed by Wareing et al. (2007c), we obtain, however, a very different cometary structure.

The model of Wareing et al. (2007c) failed to reproduce the broad-head narrow tail structure observations of Mira that Esquivel et al. (2010) matched by changing the conditions of the environment (i.e., a dense ISM is changed into a low-density rarefied medium with the parameters of the local bubble). The reason for this behavior must lie in the unrealistic temperature for the AGB wind used by Wareing et al. (2007c) $(10,000 \mathrm{~K})$ and the fact that the gas is not allowed to cool down below this value in their simulations. If we assume that the ISM temperature is the same as that used in other works by the same authors (the value is not given in Wareing et al. 2007c), then the AGB wind has always a larger temperature than the ISM. The cooling function and the temperature assumed for the wind have an important effect in the formation of the tail. Higher density regions formed behind the star will cool more efficiently and will collapse against the ISM pressure, allowing the formation of narrow tails as seen in our simulations. Narrow cometary tails are also formed in the simulations of Esquivel et al. (2010), but they require a change from a high- to a lowdensity environment. A more quantitative comparison between our models and those of Esquivel et al. (2010) is not possible given the different conditions assumed.

Although a change in the physical conditions of the ISM is possible, according to our models it is not necessary to explain the overall morphology of the Mira shell. A narrow tail is formed under our standard conditions early in the evolution of the star.

\section{DEVELOPMENT OF INSTABILITIES AND FRAGMENTATION}

Some of the computed AGB circumstellar shells are heavily fragmented; see, e.g., models $\mathrm{R}_{1 \mathrm{M}} 30 \mathrm{~h}, \mathrm{R}_{1 \mathrm{M}} 50 \mathrm{~h}$, and $\mathrm{R}_{1 \mathrm{M}} 100 \mathrm{~h}$ (also $R_{1 M} 85$ in Szentgyorgyi et al. 2003). This strong fragmentation is caused by several processes. The main cause of instabilities, as discussed extensively by Blondin \& Koerwer (1998) in their high-resolution calculations of two-dimensional and three-dimensional isothermal stellar wind bow shocks, is the nonlinear thin shell instability (NTSI; Vishniac 1994) and the transverse acceleration instability (TAI; Dgani et al. 1996) when the bow shock is radiative (nearly isothermal) in both the forward shock and the reverse shock of the stellar wind. This instability depends primarily on the Mach number of the star moving through the ISM, requiring a minimum Mach number of a few. The effect of the instability is to ripple the bow shock with wavelengths and amplitudes of the order of the nominal stand-off distance of the bow shock. Based on the internal dynamics of the slab, the overall evolution of bends in the slab, and the fact that the instability grows faster near the apex of the bow shock, Blondin \& Koerwer (1998) argued that the instability of isothermal stellar wind bow shocks could be attributed to the NTSI. The NTSI is driven by shear flows within the shell created by large-wavelength wiggles in the shell. Once the large 

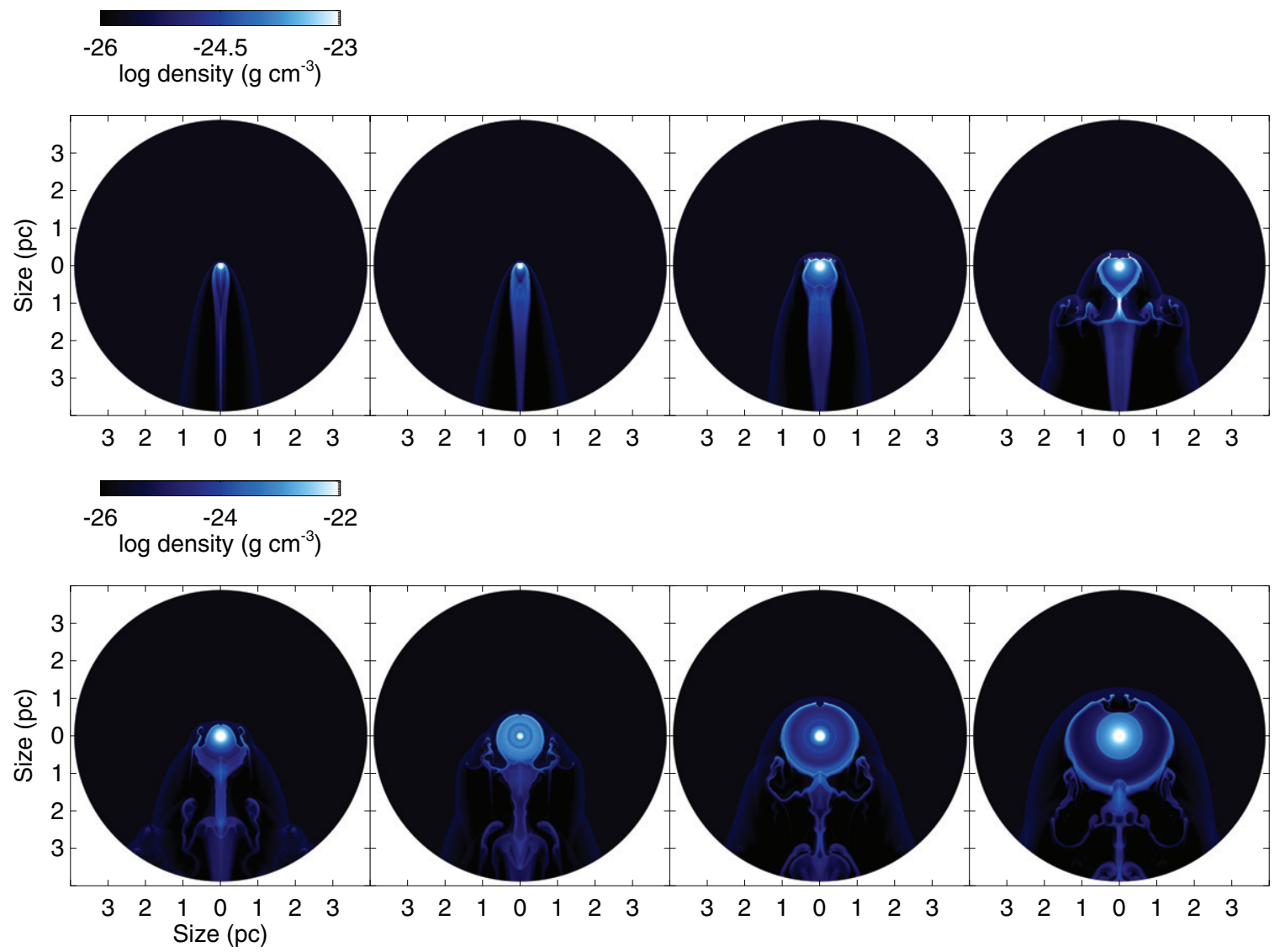

Figure 10. Same as Figure 5 but for a star moving at $100 \mathrm{~km} \mathrm{~s}^{-1}$ through an ISM with density $0.01 \mathrm{~cm}^{-3}$.

(An animation and a color version of this figure are available in the online journal.)

distortions created by the NTSI advect into the wings of the bow shock, the action of the TAI becomes important in further distorting the shape of the bow shock (see Figure 4 from Blondin \& Koerwer 1998). We believe that this is the case for models $\mathrm{R}_{1 \mathrm{M}} 30 \mathrm{~h}, \mathrm{R}_{1 \mathrm{M}} 50 \mathrm{~h}$, and $\mathrm{R}_{1 \mathrm{M}} 100 \mathrm{~h}$.

It has also been argued that red supergiant (RSG; similar to AGB in mass-loss rates and wind velocities) bow shocks are subject to Rayleight-Taylor instabilities (R-TI; Brighenti \& DErcole 1995a, 1995b; van Marle et al. 2011), since the RSG, or AGB shocked gas, and the contact discontinuity are decelerated by the shocked ISM. We think that this is the case for our model $\mathrm{R}_{1 \mathrm{M}} 1001$, as we discuss below, and not the NTSI as also discussed by Blondin \& Koerwer (1998) for the case where the forward shock is adiabatic (in our model $\mathrm{R}_{1 \mathrm{M}} 1001$, the forward shock is semi-radiative).

Wareing et al. (2007b) have also argued that the main source of instability in AGB wind bow shocks is due to the transverse shear of the flow, or the Kelvin-Helmholtz instability (K-HI). However, as it has been discussed by Blondin \& Koerwer (1998), this cannot be the case, the main reason being that the shear is only important in the wings of the bow shock. Although the resolution used by Wareing et al. (2007b) was not sufficient to claim any quantitative analysis of the instability, they argued that the K-HI was the reason for the formation of their computed vortex. Note, however, that their vortex starts near the apex of the bow shock (panels A, B, C, and D in their Figure 2), where there is no transverse shear as pointed out by Blondin \& Koerwer (1998). Note also that the shape (spiral) of the vortex is against the motion of the flow, contrary to the vortex expected by the
K-HI, where the spirals form in the direction of motion of the flow. Even more, panels C and D in their Figure 2 show an oblique shock (the vortex) piling up gas against the contact discontinuity, which is the typical behavior of the NTSI.

We then have two different scenarios in our calculations, according to the amount of radiative cooling in the forward shock. In the first one, the forward shock is radiative (similar to the isothermal case), while in the second one, the forward shock is semi-radiative. To show the difference, we can analyze models $R_{1 M} 1001$ and $R_{1 M} 100 h$, both with the same Mach number with respect to the motion in the ISM. The forward shock in the case of $\mathrm{R}_{1 \mathrm{M}} 1001$ is smooth (last panel in Figure 9) and is relatively well separated from the contact discontinuity. In this case, the fragmentation that starts in the apex is purely due to R-TI. However, the forward shock in the case $\mathrm{R}_{1 \mathrm{M}} 100 \mathrm{~h}$ (last panel of Figure 8) is corrugated, and its location is quite close to the contact discontinuity. This is due to a stronger radiative cooling in the ISM shocked gas. In both cases, the involved velocities are small, so we can assume that the bow shocks are in the radiative regime (the post-shocked gas temperature is below $10^{5} \mathrm{~K}$ ). The theory says that in these cases, the postshocked gas density should be the square of the Mach number multiplied by the ISM density (see also Blondin \& Koerwer 1998). Thus, the shocked ISM gas density in model $R_{1 M} 100 \mathrm{~h}$ would be 12.1 , while in the case of model $R_{1 M} 1001$ it would be 1.21 (see Table 1). Since the radiative cooling depends on the density as $n^{2}$, the difference in both cases is notorious. Thus, the Mach number is not the only important parameter, as discussed by Blondin \& Koerwer (1998); the density of the ISM 

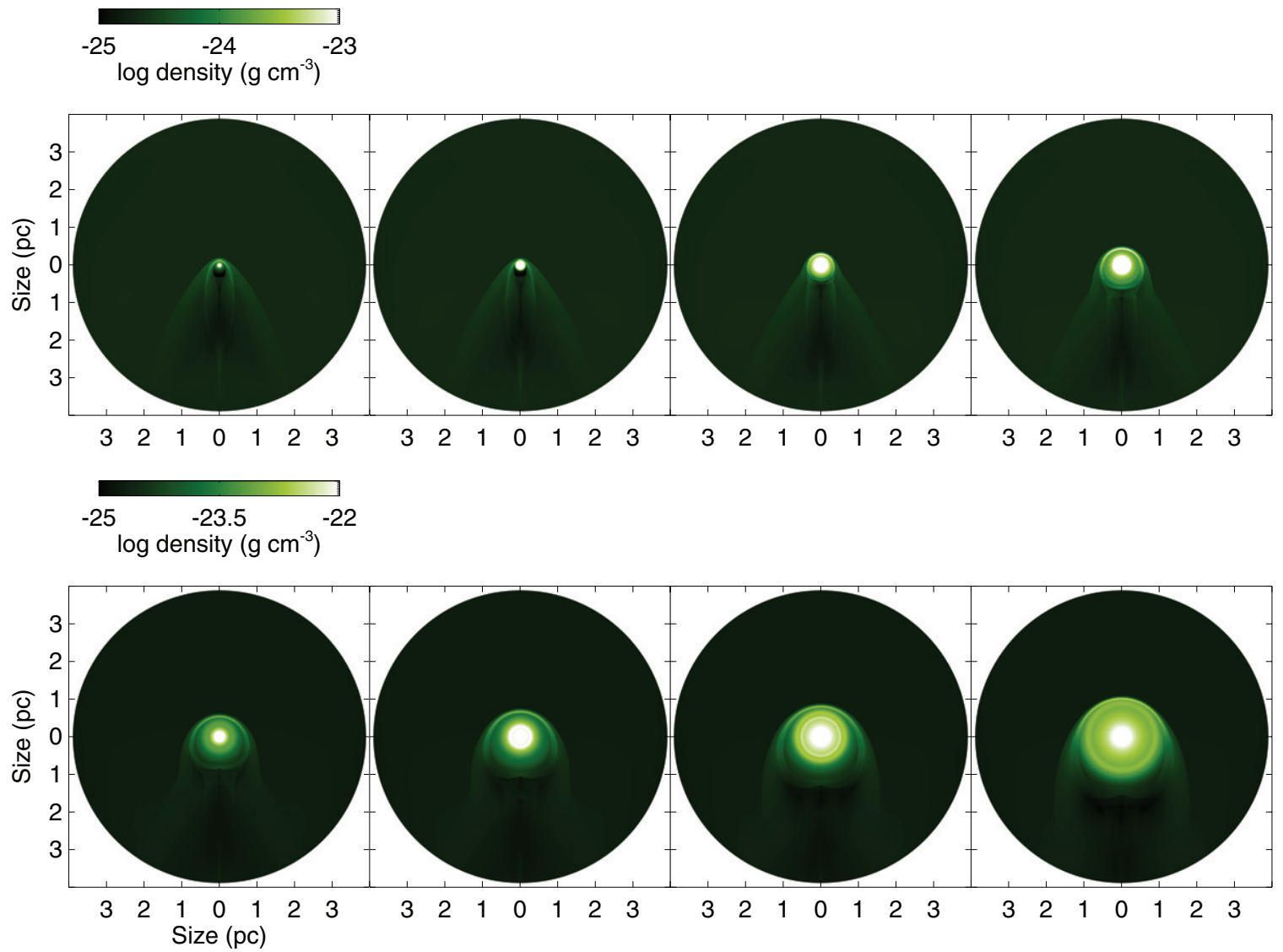

Figure 11. Density (logarithmic scale) of the circumstellar envelope of a $3.5 M_{\odot}$ AGB star moving at $20 \mathrm{~km} \mathrm{~s}^{-1}$. The ISM density is $0.1 \mathrm{~cm}{ }^{-3}$. From left to right and top to bottom, the panels show the evolution of the shell at $(1.9,2.14,2.4,2.6,2.9,3.1,3.3,3.6) \times 10^{5} \mathrm{yr}$ along the AGB. The times at which the models have been selected are marked in Figure 2, where we plot the stellar wind used in the simulations.

(An animation and a color version of this figure are available in the online journal.)

is also important. We can conclude that the case $\mathrm{R}_{1 \mathrm{M}} 1001$ is "semi-radiative" if compared with $\mathrm{R}_{1 \mathrm{M}} 100 \mathrm{~h}$ (the cooling here is 100 times larger). Thus, when the radiative cooling is strong enough (like the case of $\mathrm{R}_{1 \mathrm{M}} 100 \mathrm{~h}$ ), the forward shock is located very close to the contact discontinuity, and it is influenced by its shape, which in this case is unstable to R-TI. Once the forward shock is corrugated, the oblique shocks of the NTSI exacerbate the fragmentation of the AGB circumstellar shells.

In the cases computed here, the development of the fragmentation either by R-TI or by NTSI is exacerbated during the inter-pulse periods, since the ram pressure of the AGB wind drops considerably compared to the dynamical time.

\section{STELLAR PROGENITOR MASSES}

In Figures 11 and 12, we show the evolution of the gas density in log scale of an AGB star with initial mass 3.5 $M_{\odot}$. We have run three simulations for this stellar model (see three bottom lines of Table 1) testing two velocities for the interaction: 20 (with an ISM density of $0.1 \mathrm{~cm}^{-3}$ ) and $50 \mathrm{~km} \mathrm{~s}^{-1}$ (using ISM densities of 0.1 and $0.01 \mathrm{~cm}^{-3}$ ) covering two orders of magnitude in the value of the ram pressure. To study the process of interaction of this more massive star, we have used some of the parameters already studied for the low-mass star. In this way we better isolate the effect of the stellar mass, as reflected in the mass-loss history, in the simulations.

The first panel on the top left of the figure has been selected at $1.9 \times 10^{5} \mathrm{yr}$ into the evolution (see the first mark in Figure 2), and the subsequent panels from left to right and top to bottom have been selected every $4.8 \times 10^{3} \mathrm{yr}$. We have chosen not to show the last thousand years of the evolution given that the shell morphology, besides growing in size, does not change meaningfully from the last output (lower right corner) shown in Figures 11 and 12.

The CSEs formed at the early-AGB phase, during the constant wind period, are identical to the ones developed for the lower mass model, given that the inputs of the simulations in terms of mass-loss rate are the same during the early-AGB phase. The shape of the CSE at this early stage depends then on the parameters assumed for the interaction.

As expected, a bow-shock shape appears in the direction of the movement, but also a cometary tail is formed that is fed directly from the stellar wind and from material stripped away from the bow shock. The outermost shell departs from sphericity given that the pressure encountered by the stellar wind is not isotropic, since the stellar wind is running into a higher pressure environment in the opposite direction to the stellar movement.

Once the mass loss increases, it is kept continuously at high rates until the tip of TP-AGB. The wind expands within the cavity carved by the previously stellar wind during the whole AGB evolution of the star. Material in the bow shock is regularly deflected downstream of the movement and replenished by the stellar wind. The large amount of material contained in the outermost shell can compete effectively with the ISM ram pressure. Larger values of ram pressure (ISM relative velocity) need to be used in order to strip completely the outermost shell, 

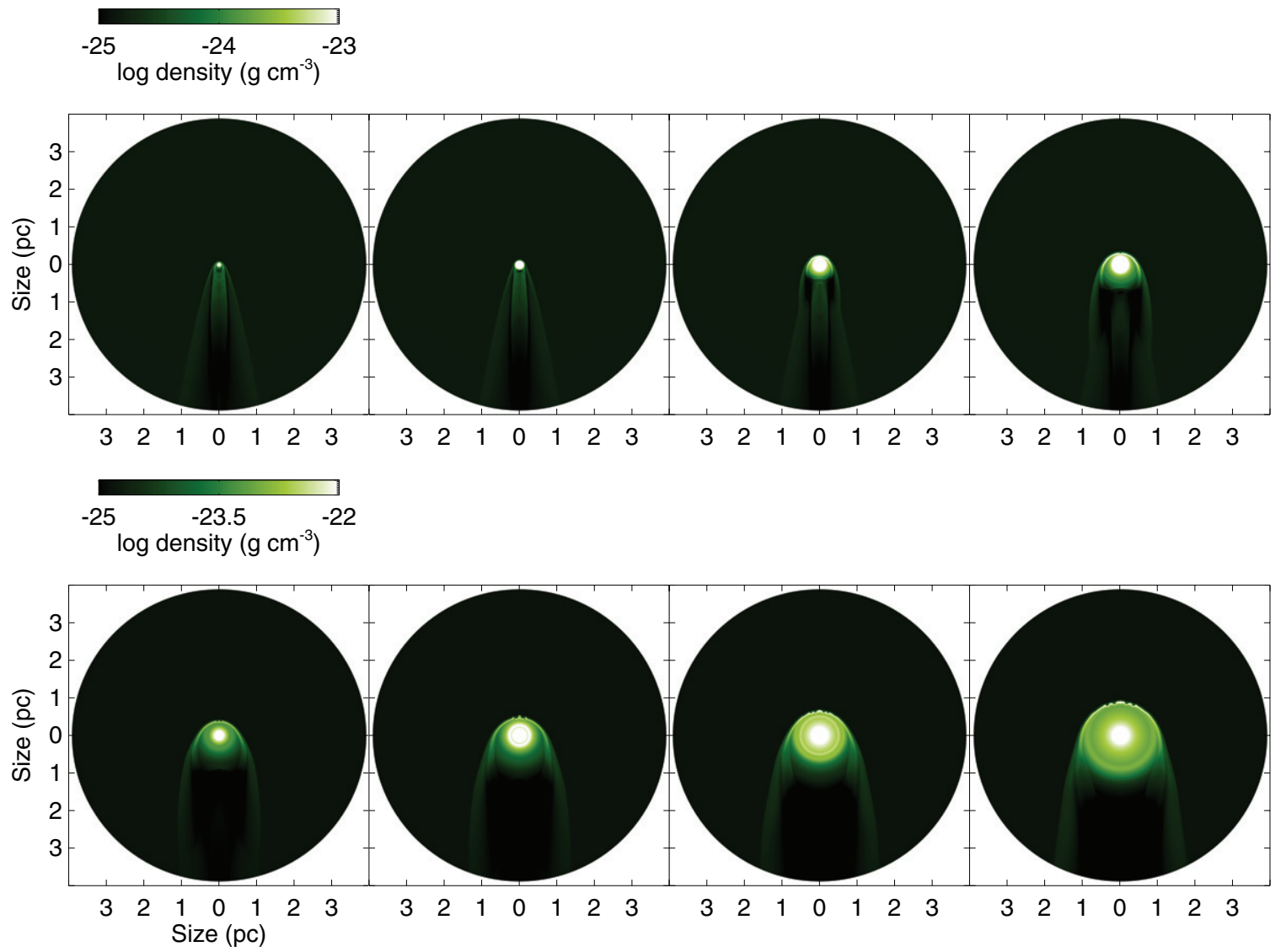

Figure 12. Same as Figure 11 but for a star moving at $50 \mathrm{~km} \mathrm{~s}^{-1}$ through an ISM with density $0.1 \mathrm{~cm}^{-3}$.

(An animation and a color version of this figure are available in the online journal.)

and therefore the freshly ejected material from the star never encounters the ISM directly. No pressure balance is reached between the CSE and the ISM. As a consequence, the shell continuously grows in the upstream direction. The wind expands in the downstream direction thorough a lower pressure cavity; no shock region develops and therefore no shell with bright edges is present.

The CSE is able to grow in size faster in the upstream direction for the same parameters of the interaction for the more massive star. The mass-loss rate history adopted in our models is constrained by the stellar evolution models of Vassiliadis \& Wood (1993). There is more than an order-of-magnitude difference in the maximum mass loss reached by the lowand high-mass stars considered $\left(0.5 \times 10^{-5}\right.$ versus $0.2 \times$ $10^{-4} M_{\odot} \mathrm{yr}^{-1}$ for the $1 M_{\odot}$ and $3.5 M_{\odot}$ star, respectively) when they reach the tip of the AGB. In addition, the mass-loss history is different, and so are the timescales of the evolution of the star in the AGB. The overall features of the interaction are the same as for the low-mass star; however, the details of the interaction differ, stressing the importance of the stellar input.

The asymmetry of the shell is barely detectable at the end of the AGB evolution in the $\mathrm{R}_{3.5 \mathrm{M}} 20 \mathrm{~h}$ model. We define $\omega$ as the ratio between the shell radius in the direction of the movement and the radius in the perpendicular direction, in order to quantify the effect of the interaction in the morphology of the shell when comparing the two models with different stellar masses. We get a value of $\omega \approx 0.85$ for the last $10^{4} \mathrm{yr}$ of the AGB evolution. For comparison $\omega=0.63$ for the $1 M_{\odot}$ model with the same parameters for the interaction (Villaver et al. 2003).
Despite the fact that relatively massive stars within the range of AGB progenitors are not expected to have a large velocity within the Galaxy, we have run simulations with an intermediate relative velocity of $50 \mathrm{~km} \mathrm{~s}^{-1}$. Model $\mathrm{R}_{3.5 \mathrm{M}} 50 \mathrm{~h}$ is shown in Figure 12. Instabilities appear in this model but do not lead to a complete destruction of the shell as they do for the same parameters of the interaction but for a low-mass star. At the end of the AGB evolution $\omega$ is 0.8 and so the disruption of the morphology is significant. Instabilities appear, but they do not have the radical influence in the morphology that they have for the low-mass model for the same parameters.

A third model for the $3.5 M_{\odot}$ star, $\mathrm{R}_{3.5 \mathrm{M}} 50 \mathrm{l}$ is listed in Table 1 . This model represents a low-density ISM $\left(0.01 \mathrm{~cm}^{-3}\right)$ with an intermediate relative velocity of $50 \mathrm{~km} \mathrm{~s}^{-1}$. The CSE has a similar evolution as the one described above, and at the end of the AGB we measure an $\omega=0.95$. The deformity in the morphology of the shell at the end of the AGB will be negligible. This is consistent with the strength of the parameters assumed for the interaction given that this model has a slightly lower ram pressure than the model $\mathrm{R}_{3.5 \mathrm{M}} 20 \mathrm{~h}$ at a lower velocity.

We find that for values of the product $n_{\mathrm{ISM}} v \approx 25-40$ or ram pressures (4-6) $\times 10^{-13}$ dyn cm$~^{-2}$ the interaction shows clearly during most of the AGB phase but disappears or will be barely noticeable during the last $10^{4} \mathrm{yr}$ of the AGB evolution and surely will not leave any imprint on the PN morphology.

\section{DISCUSSION}

The number of detected AGB stars with asymmetric shells is growing and with it the evidence that the interaction between the 
CSE and the ISM is a common phenomenon. Our models show that this is indeed the case and that the morphological features detected so far agree very well with the models presented here. Examples of the morphology of AGB stars interacting with the ISM can be found in different wavelengths and thus trace both the gas and the dust components: in $\mathrm{CO}$ and HI (see, e.g., Matthews \& Reid 2007; Matthews et al. 2008, 2011; Libert et al. 2008, 2009, 2010), in UV (Martin et al. 2007; Sahai \& Chronopoulos 2010), in the optical (Ferguson \& Ueta 2010), and in the IR (Ladjal et al. 2010; Mayer et al. 2011; Ueta et al. 2006, 2010; Ueta 2008; Jorissen et al. 2011).

A new wealth of information soon will be available on the distribution of dust around AGB stars (Groenewegen et al. 2011) and with it the possible detection of more asymmetric shells caused by the interaction with the ISM. van Marle et al. (2011) show in a simulation of a fast-moving RSG star that the dust distribution follows that of the gas when the grains considered are small, and that bigger dust grains penetrate further into the unshocked ISM. In general, for AGB stars, the size distribution of dust grains is, in principle, smooth (see, e.g., García-Hernández et al. 2007), with the stars in the lower mass range having small amounts of dust. No size segregation is, in principle, expected from different progenitor masses or dust chemical composition ( $\mathrm{C}$ versus $\mathrm{O}$-rich dust). Dust processing, however, is envisaged as the ejected AGB material mixes with the ISM non-processed matter (Villaver et al. 2002a, 2002b), as well as changes from amorphous to crystalline as the star evolves from the AGB to the PN phase (Stanghellini et al. 2007). Small grains are more abundant and have a larger collective radiative surface area, thus dominating the infrared emission (van Marle et al. 2011). Therefore, gas distributions presented in this paper should trace the dust distribution from infrared observations closely. Moreover, in the few cases where the emission at different wavelengths can be compared (RamosLarios \& Phillips 2009; Mayer et al. 2011) it seems that the distribution of dust and gas is fairly well coupled.

According to our simulations, stars observed during the earlyAGB phase, where a constant low-density wind can be assumed, are all expected to show bow-shock structures characteristic of the interaction with the ISM. This holds true for the range of conditions explored for the interaction (expanding two orders of magnitude in the value assumed for the ram pressure), and for the range of the stellar masses considered ( 1 and $\left.3.5 M_{\odot}\right)$. If this early-AGB wind lasts long enough to reach pressure equilibrium with the ISM, the location of the bow shock can be estimated analytically.

Our low-velocity models for the interaction show that, as the star ascends toward the tip of the AGB on the H-R diagram, the stellar wind always expands within the cavity created by the previous stellar wind. However, even in this case, the AGB structure is very much influenced. As already pointed out in Villaver et al. (2003), ram pressure stripping operates very efficiently in the shell interacting directly with the ISM, reducing substantially the mass of the envelope. Most of the stellar mass lost along the AGB is not to be found in the CSE; ejected mass is continuously removed and left behind the star. This is in contrast with the expectations for an AGB star at rest. If the star does not move relatively to its local ISM, the mass in the CSE will contain the amount of mass lost by the star plus a non-negligible amount of ISM mass swept out by the wind (see, i.e., Villaver et al. 2002a).

We gather that the influence of the interaction in the morphology strongly depends on the time at which the star is observed along the evolution. Early-AGB stars all show very asymmetric bow shocks along the direction of the stellar motion and collimated tails in the opposite direction. The asymmetry due to the interaction is maintained, and will be detectable, along most of the AGB for all the ram pressure conditions and stellar masses used in this work. At the end of the AGB, however, after most of the stellar mass is lost, a shell grows in size that is capable of competing with the ram pressure provided by the ISM when the stellar velocity is low $\left(10 \mathrm{~km} \mathrm{~s}^{-1}\right)$ and the stellar mass is large (and with it the mass loss). In the Galaxy, the different stellar components are characterized by different dynamical behavior. Disk stars have velocities of $10-40 \mathrm{~km} \mathrm{~s}^{-1}$ and are not expected to be found far above the Galactic plane. If we assume that the ISM gas and the stars are not moving exactly at the same speeds within the Galaxy, it is safe to expect that disk stars could be moving with respect to the ISM at low velocities (10-20 $\left.\mathrm{km} \mathrm{s}^{-1}\right)$ or, for some extreme cases, at intermediate velocities (30-50 $\mathrm{km} \mathrm{s}^{-1}$ ).

To test the validity of the application of our models, we can compare the obtained physical sizes with observed AGB stars with well-determined distances: CW Leonis (Ladjal et al. 2010), IRC +10216 (Sahai \& Chronopoulos 2010), and Mira (Martin et al. 2007). CW Leonis has a typical bow-shock structure at a distance of $0.26 \mathrm{pc}$ from the AGB star. This compares quite well with Figure 11, at a time of $3.1 \times 10^{5} \mathrm{yr}$ along the AGB (ISM density of $0.1 \mathrm{~cm}^{-3}$ and $v=20 \mathrm{~km} \mathrm{~s}^{-1}$ ). IRC +10216 has a typical bow-shock structure at a distance of $0.36 \mathrm{pc}$ from the star. This compares well with Figure 4 , at $3.4 \times 10^{5} \mathrm{yr}$ along the AGB (ISM density of $1 \mathrm{~cm}^{-3}$ and $v=10 \mathrm{~km} \mathrm{~s}^{-1}$ ). Mira shows an elongated tail of $4 \mathrm{pc}$. This compares very well with Figure 10, at $1.5 \times 10^{5} \mathrm{yr}$ along the AGB (ISM density of $0.01 \mathrm{~cm}^{-3}$ and $v=100 \mathrm{~km} \mathrm{~s}^{-1}$ ).

Regarding different stellar progenitors, our simulations show that the effects of the interaction are not noticeable in the morphology of the shells at the end of the AGB evolution of the $3.5 M_{\odot}$ star unless we consider very high values of the ISM ram pressure. The interaction, however, reduces the mass and the size of the expected shell significantly.

\subsection{Stellar Mass Loss on the AGB}

Although a full discussion regarding the treatment of mass loss during the AGB in stellar evolution calculations is beyond the scope of this work, it is in place to deliberate about the choice of the stellar evolution calculations used in our models.

Mass loss is a crucial process in the evolution of stars along the AGB; however, it cannot be calculated from first principles. High mass-loss rates (the so-called SW; Renzini 1981) are needed to remove the stellar envelope at the tip of the AGB. The scenario generally accepted nowadays for the development of these high mass-loss rates involves two entangled processes: shock waves caused by the stellar pulsation and the acceleration of dust by radiation pressure. The stellar pulsation in AGB stars creates shock waves that propagate through the stellar atmosphere. The dissipation of the mechanical energy associated with these shocks leads to the levitation of the upper layers of the atmosphere, where the gas becomes sufficiently cool (by expansion and by dilution of the stellar radiation field) and dense to allow heavy elements to condense into grains. As grains nucleate and grow, they experience the force exerted by the stellar radiation pressure and thus are accelerated. The momentum coupling between gas and dust drives the outflow. Pioneering work on dynamical calculations to drive the stellar wind was done by Wood (1979) and Bowen (1988). 
The calculation of mass loss during the AGB requires using dynamical model atmospheres in which time-dependent dynamics (shock waves and winds), radiation transfer (strong variable stellar radiation field), and dust and molecular formation processes need to be considered together. Several calculations of dust-driven winds on the AGB are available in the literature. Winters et al. (1994) models give mass-loss rates as a function of the fundamental stellar parameters for stationary atmospheres. Dynamical model atmosphere calculations have been computed by Bowen (1988), Fleischer et al. (1992), Arndt et al. (1997), and Höfner et al. (1998). Given computational limitations and our current knowledge of the fundamental physical data, it becomes clear why a full reliable prescription for mass loss on the AGB is still not available in the literature (see, e.g., Willson 2000; Willson et al. 2008). In particular, Höfner et al. (1998) demonstrated how changes in the microphysics result in considerably different mass-loss rates.

Stellar evolutionary models follow the temporal behavior of the mass loss during the AGB (Vassiliadis \& Wood 1993; Blöcker 1995; Schröder et al. 1999; Schröder \& Cuntz 2005; Wachter et al. 2002) under different prescriptions. Although the mass-loss rates are not derived from first principles in these models, and most of them rely either on the dynamical model atmosphere calculations of Bowen (1988) and Arndt et al. (1997) or on the semi-empirical mass-loss rate formula derivations of Wood (1990), they do provide a unique opportunity to study the extensive history of mass loss on the AGB and beyond. The comparison of the different mass-loss prescriptions with observations of individual stars in the AGB evolutionary phase is complicated given the variable nature of the star (and the mass loss), and a full discussion on the subject would be lengthy and beyond the scope of this work. However, it is important to note that the Vassiliadis \& Wood (1993) prescription is the most widely used and not only has not been ruled out by observations but has even been favored over other parameterizations (see, e.g., Ziljstra et al. 2002).

In our simulations, after the early-AGB phase, the star ascends the thermal-pulsing AGB where the wind is characterized by a series of SW events modulated by the thermal pulses. We have modeled the evolution of the star along this phase by constraining the stellar wind input in our simulations with the Vassiliadis \& Wood (1993) parameterization. It is important to note that the maximum mass-loss rate adopted in the Vassiliadis \& Wood (1993) models is limited to the radiation pressure limit. Dust-driven wind models allow values to be up to two times this limit. If the maximum mass-loss rates increase, the timescale of the evolution of the star is modified accordingly. By considering the evolution of the mass-loss evolution provided by a set of stellar evolution calculations, we are simulating a realistic scenario with no free parameters (note that the mass loss in other studies is arbitrarily chosen to possible AGB values).

We have taken a particular set of stellar evolution models and simulate the effects of the stellar motion in the shell formation along the AGB phase. The fine details of the evolution are expected to change under other prescriptions for the evolution of the star; however, the bulk of the results will remain for the most part unchanged. A variable wind scenario is expected for pulsating AGB stars in the thermal-pulsing phase. There are two main differences between the models presented here and the compilation by Wareing et al. (2007a): (1) the use of variable winds and different stellar masses to describe the AGB evolution of the star and (2) the use of a cooling function under $10^{4} \mathrm{~K}$ and realistic wind temperatures. Although these models represent a fair simplification of the problem, a more realistic description of the interaction requires the use of variable winds and realistic cooling functions.

\section{CONCLUSIONS}

All models, independent of the parameters used for the interaction, show prominent bow-shock structures during the early-AGB evolution. This phase is characterized by constant mass-loss rates of the order of $\sim 10^{-8} M_{\odot}$ that reach pressure equilibrium with the ISM. The morphology of the shell is a direct translation of the strength of the interaction.

Models representing a population of stars moving with relative speeds at the lower end of the velocity dispersion are characteristic of disk stars (10-20 $\mathrm{km} \mathrm{s}^{-1}$ ) and all show the same pattern. The wind always expands within the bow-shock cavity, and because it is not interacting directly with the ISM, the instabilities do not grow. Furthermore, at the tip of the AGB the stellar wind can compete with the ram pressure provided by the ISM, and the shell grows in size in the upstream direction. The fact that the wind always encounters a smaller pressure in the opposite direction of the motion causes the star to be displaced with respect the geometrical center of the envelope.

Regarding the higher end of the velocity dispersion of the disk population (30-50 $\mathrm{km} \mathrm{s}^{-1}$ ), we show that, as ram pressure stripping becomes more efficient, the stellar wind interacts directly with the ISM as the thermal pulses take place. The main consequence is the formation of instabilities in the shells. While in the upstream direction the AGB wind interacts directly with the ISM, leading to the formation of a bow shock, in the downstream direction the wind expands within the tunnel left behind by the star. Prominent elongated tails are formed in the downstream direction for these models.

We find that under the velocities expected for a population of halo stars the CSEs are expected to be instable and get fragmented. Mixing of the AGB wind with ISM material is dominant as the ISM penetrates further as the bow shock breaks up. The appearance of oblique shocks when the radiative cooling is stronger (i.e., higher ISM densities) exacerbates the fragmentation effect.

The observable effects of the interaction, although clearly visible, along most of the evolution along the AGB disappear in the morphological features at the end of the evolution for the more massive, $3.5 M_{\odot}$ models. The interaction is not expected to leave any imprint on the PN morphology for these models.

In general, ram pressure stripping is a powerful mechanism to remove mass from the envelopes of evolved stars, and even though in some cases the interaction is not expected to leave an imprint on the morphology of the envelope, its effects are important in reducing the mass and the size with respect to the values expected if the star had zero relative velocity with respect to the ISM.

We thank the anonymous referee for very relevant comments that resulted in an improvement of the original version of this paper.

\section{REFERENCES}

Arndt, T. U., Fleischer, A. J., \& Sedlmayr, E. 1997, A\&A, 327, 614

Beers, T. C., Chiba, M., Yoshii, Y., et al. 2000, AJ, 119, 2866

Binney, J., \& Merrifield, M. 1998, Galactic Astronomy (Princeton, NJ: Princeton Univ. Press)

Blöcker, T. 1995, A\&A, 297, 727

Blondin, J. M., \& Koerwer, J. F. 1998, New Astron., 3, 571 
Borkowski, K. J., Sarazin, C. L., \& Soker, N. 1990, ApJ, 360, 173 Borkowski, K. J., Tsvetanov, Z., \& Harrington, J. P. 1993, ApJ, 402, 57

Bowen, G. H. 1988, ApJ, 329, 299

Brighenti, F., \& D'Ercole, A. 1995a, MNRAS, 273, 443

Brighenti, F., \& D'Ercole, A. 1995b, MNRAS, 277, 53

Burton, W. B. 1988, in Galactic and Extragalactic Radio Astronomy, ed. K. Kellermann \& G. L. Verschuur (New York: Springer), 295

Carney, B. W., Wright, J. S., Sneden, C., et al. 1997, AJ, 114, 363

Chiba, M., \& Beers, T. C. 2000, AJ, 119, 2843

Chu, Y.-H., Gruendl, R. A., Guerrero, M. A., et al. 2009, AJ, 138, 691

Cox, D. P. 2005, ARA\&A, 43, 337

Dalgarno, A., \& McCray, R. A. 1972, ARA\&A, 10, 375

Dgani, R., van Buren, D., \& Noriega-Crespo, A. 1996, ApJ, 461, 927

Eggen, O. J., Lynden-Bell, D., \& Sandage, A. R. 1962, ApJ, 136, 748

Esquivel, A., Raga, A. C., Cantó, J., et al. 2010, ApJ, 725, 1466

Falle, S. A. E. G. 2002, ApJ, 577, L123

Ferguson, B. A., \& Ueta, T. 2010, ApJ, 711, 613

Ferrière, K. M. 2001, Rev. Mod. Phys., 73, 1031

Fleischer, A. J., Gauger, A., \& Sedlmayr, E. 1992, A\&A, 266, 321

García-Hernández, D. A., García-Lario, P., Plez, B., et al. 2007, A\&A, 462 711

Groenewegen, M. A. T., Waelkens, C., Barlow, M. J., et al. 2011, A\&A, 526, A162

Guerrero, M. A., Villaver, E., \& Manchado, A. 1998, ApJ, 507, 889

Gurzadyan, G. A. 1969, Planetary Nebulae (New York: Gordon and Breach) 235

Helmi, A. 2008, A\&AR, 15, 145

Höfner, S., Jorgensen, U. G., Loidl, R., \& Aringer, B. 1998, A\&A, 340, 497

Isaacmann, R. 1979, A\&A, 77, 327

Jorissen, A., Mayer, A., Van Eck, S., et al. 2011, A\&A, 532, A135

Kalberla, P. M. W., \& Kerp, J. 2009, ARA\&A, 47, 27

Knapp, G. R., Pourbaix, D., Platais, I., \& Jorissen, A. 2003, A\&A, 403, 993

Kulkarni, S. R., \& Heiles, C. 1988, in Galactic and Extragalactic Radio Astronomy, ed. K. Kellermann \& G. L. Verschuur (New York: Springer), 95

Ladjal, D., Barlow, M. J., Groenewegen, M. A. T., et al. 2010, A\&A, 518, L141

Libert, Y., Gérard, E., Le Bertre, T., et al. 2009, A\&A, 500, 1131

Libert, Y., Le Bertre, T., Gérard, E., \& Winters, J. M. 2008, A\&A, 491, 789

Libert, Y., Winters, J. M., Le Bertre, T., Gérard, E., \& Matthews, L. D. 2010, A\&A, 515, A112

López, J. A., García Díaz, M. T., Richer, M. G., Lloyd, M., \& Meaburn, J. 2011, arXiv:1101.5653

MacDonald, J., \& Bailey, M. E. 1981, MNRAS, 197, 995

Mac Low, M.-M., van Buren, D., Wood, D. O. S., \& Churchwell, E. 1991, ApJ, 369,395

Majewski, S. R. 1993, ARA\&A, 31, 575

Martin, D. C., Seibert, M., Neill, J. D., et al. 2007, Nature, 448, 780

Matthews, L. D., Libert, Y., Gérard, E., Le Bertre, T., \& Reid, M. J. 2008, ApJ, 684, 603

Matthews, L. D., Libert, Y., Gérard, E., et al. 2011, AJ, 141, 60

Matthews, L. D., \& Reid, M. J. 2007, AJ, 133, 2291

Mayer, A., Jorissen, A., Kerschbaum, F., et al. 2011, A\&A, 531, L4

McKee, C. F., \& Ostriker, J. P. 1977, ApJ, 218, 148

Raga, A. C., \& Cantó, J. 2008, ApJ, 685, L141

Raga, A. C., Cantó, J., De Colle, F., et al. 2008, ApJ, 680, L45
Ramos-Larios, G., \& Phillips, J. P. 2009, MNRAS, 400, 575

Ransom, R. R., Uyaniker, B., Kothes, R., \& Landecker, T. L. 2008, ApJ, 684, 1009

Raymond, J. C., \& Smith, B. W. 1977, ApJS, 35, 419

Renzini, A. 1981, in Proc. Second Workshop on Physical Processes in Red Giants, Erice, Italy, ed. I. Iben, A. Renzini, \& D. Dordrech (Dordrecht: Reidel), 431-446

Ryde, N., \& Schöier, F. L. 2001, ApJ, 547, 384

Sahai, R., \& Chronopoulos, C. K. 2010, ApJ, 711, L53

Schönberner, D., Jacob, R., Steffen, M., et al. 2005, A\&A, 431, 963

Schröder, K.-P., \& Cuntz, M. 2005, ApJ, 630, L73

Schröder, K.-P., Winters, J. M., \& Sedlmayr, E. 1999, A\&A, 349, 898

Smith, H. 1976, MNRAS, 175, 419

Soker, N., Borkowski, K. J., \& Sarazin, C. L. 1991, AJ, 102, 1381

Soker, N., \& Zucker, D. B. 1997, MNRAS, 289, 665

Stanghellini, L., García-Lario, P., García-Hernández, D. A., et al. 2007, ApJ, 671,1669

Stone, J. M., Mihalas, D., \& Norman, M. L. 1992, ApJS, 80, 819

Stone, J. M., \& Norman, M. L. 1992a, ApJS, 80, 753

Stone, J. M., \& Norman, M. L. 1992b, ApJS, 80, 791

Szentgyorgyi, A., Raymond, J., Franco, J., Villaver, E., \& López-Martín, L. 2003, ApJ, 594, 874

Tweedy, R. W., \& Kwitter, K. B. 1996, ApJS, 107, 255

Tweedy, R. W., Martos, M. A., \& Noriega-Crespo, A. 1995, ApJ, 447, 257

Ueta, T. 2008, ApJ, 687, L33

Ueta, T., Speck, A. K., Stencel, R. E., et al. 2006, ApJ, 648, L39

Ueta, T., Stencel, R. E., Yamamura, I., et al. 2010, A\&A, 514, A16

van Buren, D., Mac Low, M.-M., Wood, D. O. S., \& Churchwell, E. 1990, ApJ, 353,570

van Marle, A. J., Meliani, Z., Keppens, R., \& Decin, L. 2011, ApJ, 734, L26

Vassiliadis, E., \& Wood, P. 1993, ApJ, 413, 641

Villaver, E., García-Segura, G., \& Manchado, A. 2002a, ApJ, 571, 880

Villaver, E., García-Segura, G., \& Manchado, A. 2003, ApJ, 585, L49

Villaver, E., Manchado, A., \& García-Segura, G. 2002b, ApJ, 581, 1204

Villaver, E., \& Stanghellini, L. 2005, ApJ, 632, 854

Vishniac, E. T. 1994, ApJ, 428, 186

Wachter, A., Schröder, K.-P., Winters, J. M., Arndt, T. U., \& Sedlmayr, E. 2002, A\&A, 384, 452

Wareing, C. J., O'Brien, T. J., Zijlstra, A. A., et al. 2006, MNRAS, 366, 387

Wareing, C. J., Zijlstra, A. A., \& O'Brien, T. J. 2007a, MNRAS, 382, 1233

Wareing, C. J., Zijlstra, A. A., \& O'Brien, T. J. 2007b, ApJ, 660, L129

Wareing, C. J., Zijlstra, A. A., O’Brien, T. J., \& Seibert, M. 2007c, ApJ, 670, L125

Willson, L. A. 2000, ARA\&A, 38, 573

Willson, L. A., Struck, C., Wang, Q., \& Kawaler, S. D. 2008, Phys. Scr., 133, 014008

Winters, J. M., Dominik, C., \& Sedlmayr, E. 1994, A\&A, 288, 255

Wood, P. R. 1979, ApJ, 227, 220

Wood, P. R. 1990, in From Miras to Planetary Nebulae: Which Path for Stellar Evolution?, ed. M. O. Mennessier \& A. Omont (Yvette Cedex: Éditions Frontières), 67

Xilouris, K. M., Papamastorakis, J., Paleologou, E., \& Terzian, Y. 1996, A\&A, 603,612

Ziljstra, A. A., Bedding, T. R., \& Mattei, J. A. 2002, MNRAS, 334, 498 